\title{
Ezh2 regulates the Lin28/let-7 pathway to restrict activation of fetal gene signature in
} adult hematopoietic stem cells

Motohiko Oshima ${ }^{1,2}$, Nagisa Hasegawa ${ }^{1}$, Makiko Mochizuki-Kashio ${ }^{1,2}$, Tomoya Muto ${ }^{1}$, Satoru Miyagi ${ }^{1,2}$, Shuhei Koide ${ }^{1,2}$, Shogo Yabata $^{1}$, George Russel Wendt ${ }^{1}$, Atsunori Saraya ${ }^{1,2}$, Changshan Wang ${ }^{1,2,3}$, Kazuya Shimoda ${ }^{4}$, Yutaka Suzuki ${ }^{5}$, Atsushi Iwama ${ }^{1,2}$

1. Department of Cellular and Molecular Medicine, Graduate School of Medicine, Chiba University, Chiba 260-8670, Japan

2. JST, CREST, Gobancho, Chiyoda-ku, Tokyo, Japan

3. College of Life Sciences, Inner Mongolia University, Hohhot 010021, China.

4. Department of Gastroenterology and Hematology, Faculty of Medicine, University of Miyazaki, Miyazaki 889-1692, Japan

5. Laboratory of Functional Genomics, Department of Medical Genome Sciences, Graduate School of Frontier Sciences, University of Tokyo 102-0075, Japan

Corresponding author:

Atsushi Iwama, M.D. Ph.D. (aiwama@faculty.chiba-u.jp)

Department of Cellular and Molecular Medicine, Graduate School of Medicine, Chiba University, 1-8-1 Inohana, Chuo-ku, Chiba 260-8670, Japan.

Phone: +81-43-226-2187, Fax: +81-43-226-2191

The total number of words: 4,781 


\begin{abstract}
Fetal liver hematopoietic stem cells (HSCs) seed bone marrow (BM) and undergo reprograming into adult type HSCs that are largely quiescent and restricted in their self-renewal activity. Here we show that in the absence of the polycomb-group gene Ezh2, a cohort of fetal-specific genes, including let-7 target genes, were activated in BM hematopoietic stem and progenitor cells (HSPCs), leading to acquisition of fetal phenotypes by BM HSPCs, such as enhanced self-renewal activity and production of fetal-type lymphocytes. The Lin28b/let-7 pathway determines developmentally timed changes in HSPC programs. Of note, many of the fetal-specific let-7 target genes, including Lin28, appear to be transcriptionally repressed by Ezh2-mediated H3K27me3 in BM HSPCs, and Ezh2 loss results in their ectopic expression, particularly in hematological malignancies that develop in the absence of Ezh2. These findings suggest that Ezh2 cooperates with let-7 microRNAs in silencing the fetal gene signature in BM HSPCs and restricts their transformation.
\end{abstract}

\title{
Highlights
}

Ezh2 loss results in activation of fetal gene signature in adult mouse BM HSPCs Adult BM HSPCs reacquire fetal phenotypes upon Ezh2 loss Ezh2 directly represses fetal-specific let-7 target genes such as Lin28b in BM HSPCs Ezh2 cooperates with let-7 microRNAs in silencing fetal gene signatures in BM HSPCs

\section{Key words:}

Hematopoietic stem and progenitor cells; Ezh2; fetal signature; let-7; Lin28 


\section{Introduction}

Hematopoietic stem cells (HSCs) first appear around embryonic day 10.5 (E10.5) in mice and seed in the fetal liver (FL) that serves as the main hematopoietic organ during the fetal period $(1,2)$. During fetal development, fetal HSCs undergo rapid self-renewal and increase their numbers to meet the demand of development. After birth, HSCs in the bone marrow (BM), which are predominantly quiescent, maintain hematopoietic homeostasis throughout life (3-5). FL HSCs transplanted into adult recipient mice show faster expansion and greater repopulating capacity compared with adult HSCs, and then acquire adult characteristics after 6 weeks $(3,5,6)$. The phenotypic difference between fetal and adult HSCs could be partially caused by different niches. However, gene expression profiling of HSCs has also revealed a role of cell-autonomous mechanisms $(7,8)$ and several genes have been demonstrated to be essential specifically for either fetal HSCs $(\operatorname{Sox} 17, \operatorname{Lin} 28 b)(9-11)$ or adult HSCs (Bmi1, Cebpa and let-7 microRNAs) (9, 12-14). Lin28b encodes an RNA-binding protein that blocks the maturation of let-7 microRNAs. Of interest, Lin $28 b$ itself is regulated by let- 7 microRNAs. Thus, Lin28b and let-7 function as a molecular switch. Expression of Lin28b and let-7 microRNAs is mutually exclusive, and the Lin28b/let-7-Hmga2 axis plays a critical role in controlling developmental changes in HSC properties (9) and neural stem cells (15). Many of the let-7 target genes, such as Hmga2, $K$-Ras, $c$-Myc and Igfbp3, have been characterized as 'oncofetal' genes, or genes that are highly expressed in early embryogenesis and reactivated in various tumors (16-18).

Polycomb group (PcG) protein Ezh2 is an enzymatic component of polycomb repressive complex (PRC) 2, which participates in gene silencing by catalyzing trimethylation of histone $\mathrm{H} 3$ at lysine 27 (H3K27me3). We previously reported that Ezh2 is indispensable for FL hematopoiesis but largely dispensable for adult BM hematopoiesis (19). However, FL hematopoietic cells transplanted into adult recipients showed greater 
reconstitution capacity and established highly myeloid-biased reconstitution compared with wild-type (WT) hematopoietic cells when Ezh2 was deleted specifically in donor cells after engraftment in the BM. Furthermore, Ezh2-deficient hematopoietic cells eventually induced myelodysplastic/myeloproliferative neoplasm (MDS/MPN)-like disorders characterized by myelodysplasia with enhanced proliferative capacity of HSCs in recipient mice (20). These findings correspond well with the identification of inactivating EZH2 somatic mutations, which often coincide with somatic mutations in genes such as TET2, in myelodysplastic disorders (20-23). Indeed, we have shown that concurrent depletion of Ezh2 and Tet2 markedly accelerates the development of myelodysplastic syndrome (MDS) and MDS/MPN in mice (20). Based on these findings, we hypothesized that upon Ezh2 loss, adult BM HSCs re-acquire some features characteristic of fetal HSCs, which show greater repopulation capacity and biased differentiation compared with adult BM HSCs.

To address this question, we performed gene expression profiling combined with ChIP-sequencing of fetal, adult and Ezh2-deficient hematopoietic stem and progenitor cells (HSPCs) and confirmed that Ezh2 loss causes sustained activation of a fetal-specific gene signature including the Lin28b/let-7 pathway genes in adult BM HSPCs, which may accounts for sustained fetal-like high self-renewal capacity and the propensity of Ezh2-deficient HSCs to undergo malignant transformation. 


\section{Results}

\section{Ezh2-deficient HSPCs retain fetal-like gene signature}

In our previous study (20), we transplanted E14.5 fetal liver cells from Cre-ERT control, Cre-ERT;Ezh $2^{f l / f l}$ or Cre-ERT;Ezh $2^{f l f l} T e t 2^{K D / K D}$ mice (CD45.2) into lethally irradiated CD45.1 recipient mice. After engraftment, we then deleted $E z h 2$ by intraperitoneal injection of tamoxifen at 4 weeks post-transplantation (hereafter refer to as WT, $E z h 2^{4 / \Delta}$ and

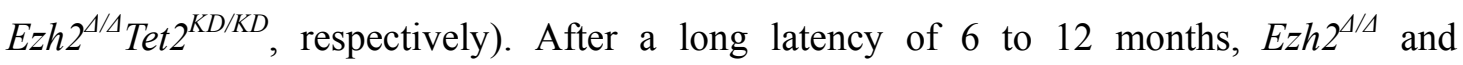
$E z h 2^{\Delta / \Delta} T e t 2^{K D / K D}$ mice developed MDS or MDS/MPN. We purified WT and $E z h 2^{\Delta / \Delta}$ Lineage ${ }^{-S c a}-1^{+} \mathrm{c}-\mathrm{Kit}^{+}$(LSK) HSPCs and granulocyte-macrophage progenitors (GMPs) from the recipient mice at 3 months post-deletion of Ezh2 (pre-disease stage) and subjected them to microarray analysis (LSK cells and GMPs) and ChIP-sequencing of the H3K27me3 modification (GMPs). Ezh2 $2^{4 / \Delta} \operatorname{Tet} 2^{K D / K D}$ LSK cells were also purified from the recipient mice that developed MDS or MDS/MPN and subjected to microarray analysis (Fig. 1, left panel). To understand the fetal-like phenotypes of Ezh2-deficientBM HSPCs, we compared above-mentioned published data with newly obtained data of microarray analysis (LSK cells) and ChIP-sequencing of H3K27me3 (LSK cells and GMPs) in WT cells from E15.5 FL and adult BM (8 weeks old) (Fig. 1, right panel).

Among NCBI reference sequence (RefSeq) genes, 453 genes were upregulated (more than 2-fold or 5-fold for genes which became transcriptionally detectable from undetectable levels) in $E z h 2^{\Delta / \Delta}$ BM LSK cells compared with WT (hereafter refer to as " $E z h 2^{\Delta / \Delta} \mathrm{LSK}$ UP genes") (Fig. 2A). In FL LSK cells 1,130 genes were expressed greater than 2-fold compared with adult BM LSK cells (or 5-fold for genes which are transcriptionally undetectable in BM LSK cells but are detectable in fetal liver LSK cells) (hereafter refer to as "FL-specific genes")(Fig. 2A). Of interest, 101 genes (22\% of $E z h 2^{4 / \Delta}$ LSK UP genes and $9 \%$ of FL-specific genes) overlapped between these two gene sets ( $\mathrm{p}$-value $\left.<1.0 \times 10^{-16}\right)$ (Fig. 
2B and Supplementary Table E1). Gene set enrichment analysis (GSEA) also showed that FL-specific genes are positively enriched with statistical significance in $E z h 2^{4 / \Delta}$ BM LSK cells (Fig. 2C). Correspondingly, FL-specific genes tended towards up-regulation in $E z h 2^{4 / \Delta}$ BM LSK cells compared with WT $(\mathrm{R}=0.142)$ (Fig. 2D). These findings indicate that transcriptional signature specific to FL HSPCs is at least partially activated in BM HSPCs in the absence of Ezh2.

We then compared the gene expression profile of $E z h 2^{4 / \Delta}$ BM LSK cells with those of $\mathrm{BM}$ CD150 ${ }^{+} \mathrm{CD} 48^{-}$LSK HSCs deficient for $E e d$, which encodes a core component of PRC2, and Ezhl, which encodes another enzymatic component of PRC2, and of BM LSK HSPCs deficient for Bmil, which encodes a core component of PRC1 (24-26). GSEA revealed that the $E z h 2^{4 / \Delta}$ LSK UP genes are positively enriched with statistical significance in $\mathrm{Eed}^{4 / \Delta} \mathrm{BM}$ HSCs but not in $E z h 1^{\Delta / \Delta}$ BM HSCs or Bmil $1^{-/}$LSK cells (Fig. 2E). Notably, however, FL-specific genes were positively enriched with statistical significance in all HSCs/HSPCs deficient for PcG genes (Eed, Ezh1 and Bmil) as in Ezh2 $2^{4 / 4}$ BM HSPCs (Fig. 2F). Furthermore, gene expression signatures of HSPCs from yolk sac, placenta, aorta-gonad mesonephros (AGM) and fetal liver (7) were all positively enriched with statistical significance in $E z h 2^{4 / \Delta}$ BM HSPCs (Fig. 2G). These findings suggest that dysregulated function of PcG complexes causes activation of a fetal-specific gene signature in adult BM HSPCs. This finding was further confirmed by comparison of $E z h 2^{4 / 4} \mathrm{BM}$ HSPCs with BM hematopoietic progenitor cells (HPCs) overexpressing Sox17. Sox17 is an essential regulator of fetal HSCs and when overexpressed in BM CD48 ${ }^{+}$LSK HPCs, it activates a set of fetal HSC genes (Fig. 2F) $(10,11) . E z h 2^{4 / \Delta}$ LSK UP genes were positively enriched with statistical significance in BM HPCs overexpressing Sox17 (Fig. 2E), further supporting the fetal-like gene signature in BM HSPCs in the absence of Ezh2. 


\section{De-repression of let-7 target genes in Ezh2-deficient adult BM HSPCs}

Among fetal-specific HSC regulators, Lin28b is exclusively expressed in fetal HSPCs. Lin $28 \mathrm{~b}$ blocks the maturation of let-7 microRNAs, thereby up-regulates expression of let-7 target genes. Ectopic expression of Lin28b reprograms adult BM HSPCs to gain augmented self-renewal capacity. Among let-7 targets, Hmga2 has been attributed to augmentation of self-renewal capacity of fetal HSCs (9). Changes in let-7 and Hmga2 expression also determine developmental stage-specific functions of neural stem cells (15). These findings clearly indicate that the Lin28b/let-7 pathway plays a critical role in controlling developmental changes in stem cell properties (9). As reported, Lin28b was detected only in fetal liver HSPCs while let-7b and let-7c were preferentially expressed in adult BM HSPCs (Fig. 3A). Of note, Lin28b was significantly de-repressed in $E z h 2^{4 / 4}$ BM LSK cells and myeloid progenitors, including GMPs and erythroblast-megakaryocyte progenitors (MEPs), compared with WT BM LSK cells, although its expression levels were much lower compared with its physiological levels in FL HSPCs (approximately 10-fold less than that in FL HSPCs) (Fig. 3B). Accordingly, let7b and let-7c were mildly but significantly downregulated in $E z h 2^{4 / \Delta}$ BM LSK cells and myeloid progenitors compared with WT cells (Fig. 3B). As Lin28b itself is one of the let-7 target genes, we then focused on the expression of other let-7 target genes. Within 1,648 putative let-7 target genes, 134 genes were highly expressed in FL LSK cells (more than 2-fold or 5-fold for genes which became transcriptionally detectable from undetectable levels) (hereafter refer to as "FL-specific let-7 target genes”) (Fig. 3C and Supplementary Table E2). These FL-specific let-7 target genes showed a clear trend towards up-regulation in $E z h 2^{4 / \Delta}$ BM LSK cells compared with WT cells $(\mathrm{R}=0.258)$ (Fig. 3C) in a manner similar to FL-specific genes (Fig. 3D). GSEA revealed significant enrichment of FL-specific let-7 target genes in $E z h 2^{\Delta / \Delta}$ BM LSK cells and GMPs compared with WT cells (Fig. 3E). FL-specific let-7 target genes were also 
positively enriched with statistical significance in HSCs/HSPCs deficient for PcG genes (Eed and Bmil) but not in HPCs overexpressing Sox17 (Fig. 3F). RT-PCR analysis confirmed de-repression of representative let-7 targets such as $\mathrm{Hmga2}$ and $\operatorname{Igf} 2 \mathrm{bp} 3$ in $E z h 2^{4 / \Delta}$ BM LSK cells and myeloid progenitors. Notably, their expression levels were comparable to their physiological expression levels in FL HSPCs (Figs. 3A,B). These findings clearly demonstrate that many of the fetal-specific let-7 target genes that control fetal-specific HSC properties are de-repressed in Ezh2 $2^{4 / \Delta}$ HSPCs.

\section{Active fetal-like lymphopoiesis by adult BM HSPCs upon Ezh2 loss}

The initial wave of lymphoid development mediated by fetal HSPCs is biased towards the production of fetal-type lymphocytes, such as B-1a B cells, marginal zone (MZ) B cells, and $\gamma \delta$ T cells. Although the genetic programs that control distinct stem cell fates remain poorly understood, Lin28b, which is specifically expressed in the fetal liver and thymus, but not in adult BM and thymus, has been reported to reprogram adult BM HSPCs to initiate fetal-like lymphopoiesis $(27,28)$. Fetal liver B-1 cells are innate-like lymphocytes that generate natural polyreactive IgM antibodies with important functions in tissue homeostasis and immune defense. B-1 cells, which include $\mathrm{CD}^{+} \mathrm{B}-1 \mathrm{a}$ and $\mathrm{CD}^{-} \mathrm{B}-1 \mathrm{~b}$ cells, are found at relatively high frequencies within peritoneal and pleural cavities of mice (29). MZ B cells, so named because of their localization in the splenic follicle, are functionally similar to B-1 cells in that they secrete $\operatorname{IgM}$ antibodies in response to blood-borne pathogens in a $\mathrm{T}$ cell-independent manner. FL but not adult BM is an efficient source of B-1a and MZ B cells, and fetal liver HSPCs preferentially produce B-1a and MZ B cells upon transplantation, unlike adult BM HSPCs $(27,30,31)$. Of interest, at 3 months post-deletion of Ezh2 in the BM, the majority of the $E z h 2^{4 / 4}$ mice showed dramatic expansion of $\mathrm{CD} 19^{+} \mathrm{B} 220^{-/ 1 \text { low }} \mathrm{CD}^{+} \mathrm{B}-1 \mathrm{a} \mathrm{B}$ cells in the peritoneal cavity (Fig. 4A). B220 ${ }^{+} \mathrm{CD} 1 \mathrm{~d}^{+} \mathrm{CD} 23^{-} \mathrm{MZ}$ B cells in the spleen were 
also increased 3-fold in $E z h 2^{4 / \Delta}$ mice compared with the control mice (Fig. 4B). Conversely, the proportions of conventional B-2 B cells $\left(\mathrm{CD} 19^{+} \mathrm{B} 220^{+} \mathrm{CD} 5^{-}\right)$and follicular $(\mathrm{FO}) \mathrm{B}$ cells $\left(\mathrm{B} 220^{+} \mathrm{CD} 1 \mathrm{~d}^{-} \mathrm{CD} 23^{+}\right)$were decreased in the peritoneal cavity and the spleen, respectively, compared with WT mice (Figs. 4A,B). Furthermore, $E z h 2^{4 / \Delta}$ mice produced 50 -fold more $\gamma \delta$ $\mathrm{T}$ cells $\left(\mathrm{CD}^{+} \mathrm{TCR} \gamma \delta^{+}\right)$in the thymus (Fig. 4C). These data clearly indicate that a fetal-specific gene program promotes fetal-type lymphopoiesis by adult BM HSPCs in the absence of Ezh2.

\section{Ezh2 keeps let-7 target genes transcriptionally repressed in adult BM}

In order to evaluate whether Ezh2 directly targets fetal-specific genes in adult BM, we next performed ChIP-sequence analysis of H3K27me3 levels in WT FL and adult BM LSK cells

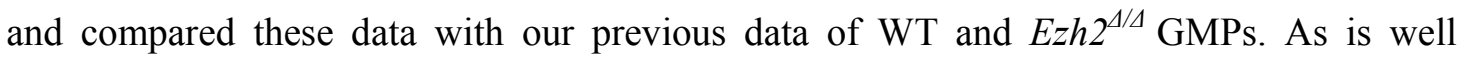
recognized, the levels of $\mathrm{H} 3 \mathrm{~K} 27 \mathrm{me} 3$ at promoter regions (TSS $\pm 2.0 \mathrm{~kb}$ of RefSeq genes) showed an inverse correlation with expression levels (Fig. 5A). Correspondingly, FL-specific genes and FL-specific let-7 target genes had lower levels of H3K27me3 at their promoters in FL cells compared with BM cells (Fig. 5B). Among all RefSeq genes, 503 genes acquired the H3K27me3 modification ( $\geq 2$-fold increase) in adult BM LSK cells compared with FL LSK cells, and thus were considered as canonical direct targets of Ezh2 specific to adult BM HSPCs (hereafter refer to BM-specific PRC2 target genes) (Fig. 5C). Of note, $16.1 \%$ of them ( 81 genes) were FL-specific genes (Fig. 5C and Supplementary Table E3), indicating that a significant portion of fetal-specific genes is silenced in adult $\mathrm{BM}$ via $\mathrm{H} 3 \mathrm{~K} 27 \mathrm{me} 3$ modification. This trend was also true with FL-specific let-7 target genes. Among all let-7 target genes, 44 genes underwent a $\geq 2$-fold increase in the levels of H3K27me3 in adult BM LSK cells compared with FL LSK cells, and $38.6 \%$ of these genes (17 genes) were FL-specific let-7 target genes (Fig. 5D and Supplementary Table E3). 
We next checked the changes in H3K27me3 levels at promoters of FL-specific and FL-specific let-7 target genes using our previous ChIP-sequence data of $E z h 2^{4 / \Delta}$ GMPs in adult BM (20). Upon deletion of Ezh2, a large number of genes experienced reduced H3K27me3 levels at their promoters in GMPs, and this reduction was more evident with FL-specific genes and even more evident with FL-specific let-7 target genes compared with all genes (Figs. 6A,B). Upon reduction in H3K27me3 levels, both FL-specific genes and FL-specific let-7 target genes showed a clear trend toward de-repression in BM LSK cells (Fig. 6C). Many of the 17 FL-specific let-7 targets, which underwent H3K27me3 modification in adult BM (Fig. 5D), were de-repressed in the absence of Ezh2 in BM LSK cells, including Lin28b, Hmga2, Jam3, Igf2bp3, Lphn1 and Zfp354a (Figs. 6C,D). These results indicate that FL-specific let-7 target genes are negatively regulated not only by let-7 microRNAs at the post-transcriptional level, but also by Ezh2-mediated H3K27me3 at the transcriptional level in adult BM HSPCs.

\section{Escape of let-7 target genes from Ezh2-mediated silencing is associated with progression of hematological malignancies}

Many let-7 target genes, including Lin28b, Igfbp3 and Hmga2 are characterized as 'oncofetal' genes, or genes that are highly expressed in early embryogenesis but reactivated in various tumors $(16,17)$. We therefore asked whether the expression of let-7 targets in LSK cells is associated with progression of hematological malignancies. As in Figure 1, we have previously shown that deletion of Ezh2 alone or concurrent depletion of Ezh2 and Tet2 induces MDS and MDS/MPN-like disorders in mice (20). We re-analyzed our previous microarray data of LSK cells from $E z h 2^{\Delta / A} T e t 2^{K D / K D}$ mice at pre-disease stage (3 months post-deletion of Ezh2) and with MDS or MDS/MPN. In line with the findings in this study, FL-specific genes as well as FL-specific let-7 target genes were significantly enriched in 
LSK cells from $T e t 2^{K D / K D} E z h 2^{4 / \Delta}$ mice compared with those from WT mice at 3 months post-deletion of Ezh2, and of note, these genes became further enriched in MDS and MDS/MPN mice (Figs. 7A,B). Arid3a and Bcl2ll/Bclx are such genes that showed clear upregulation in MDS and MDS/MPN compared with the earlier time points post-deletion of Ezh2 (Fig. 7B). Of interest, however, Arid $3 a$ and Bcl2l1/Bclx are not direct targets of Ezh2 in BM HSPCs, suggesting that even the let-7 target genes not regulated by Ezh2 could be activated in the absence of Ezh2 in BM HSPCs.

We then checked the expression levels of let-7 target genes in human MDS patients (32). Interestingly, FL-specific let-7 target genes showed a trend towards positive enrichment in $\mathrm{CD} 4^{+}$cells from MDS patients, particularly from MDS patients with 7q deletion, which at a high frequency results in the loss of EZH2 at 7q36.1 (Fig. 7C). Among these, several genes, such as IGF2BP2,IGF2BP3, ARID3A, JAM3 and LPHN1 showed significantly higher expression in $\mathrm{CD}^{+} 4^{+}$cells from MDS patients with $7 \mathrm{q}$ deletion compared with healthy controls (Fig. 7D). Although the expression of $L I N 28 B$ and HMGA2 in MDS CD34 ${ }^{+}$ cells was comparable to healthy cells, HMGA2 and LET7 microRNAs (LET7A1 and LET7C) are reported to be significantly upregulated and downregulated, respectively, in $\mathrm{CD} 4^{+}$cells from patients with primary myelofibrosis $(33,34)$, the most severe type of MPN that often harbors inactivating mutations in PRC2 genes including EZH2 (21,22). Together, these results support the notion that de-repression of oncofetal let-7 target genes by Ezh2 insufficiency contributes to the pathogenesis of hematopoietic disorders. 


\section{Discussion}

In this study, we demonstrated that Ezh2 deficiency causes activation of a fetal gene signature in BM HSPCs partly through deregulation of the Lin28b/let-7 pathway genes. Given that this trend is highly conserved in Eed-deficient HSCs and Bmil-deficient HSPCs, the canonical PcG complexes may play a role in silencing fetal-specific genes in BM HSPCs. Let-7 expression has been noted as the key determinant that represses expression of let-7 target genes in adult BM HSPCs $(9,27)$. However, our findings uncovered that FL-specific let-7 target genes are repressed not only by let-7 microRNAs at the post-transcriptional level, but also by Ezh2-mediated H3K27me3 at the transcriptional level in adult BM HSPCs (Fig. 8). Lin28b, one of FL-specific let-7 targets and the inhibitor of let-7 miRNA biogenesis, was de-repressed and caused mild reduction in let-7 levels in $E z h 2^{4 / \Delta}$ BM LSK cells, thereby further promoting de-repression of FL-specific let-7 target genes. However, not all the FL-specific Let-7 targets marked by H3K27me3 specifically in the BM became transcriptionally active despite significant reduction in $\mathrm{H} 3 \mathrm{~K} 27$ me levels at their promoters. This could be due to the lack of FL-specific transcriptional activators acting on their promoters and enhancers that are essential for the full activation of fetal transcriptional program. Another possible cause is the lack of inputs from the fetal niche. Nonetheless, modification of these FL-specific genes with H3K27me3 could play a physiologically significant role in restricting their activation in response to unfavorable intrinsic and extrinsic insults. Interestingly, FL-specific genes but not FL-specific let-7 target genes showed close association with Sox 17 expression (Figs. 2F, 3E), suggesting that the Lin28b/Let-7 and Sox17 pathways operate independently and target different genes in the transcriptional regulation of fetal-specific gene signature. In adult BM, however, Ezh2 targets both pathways to repress fetal-specific gene signature as demonstrated in Figure 2. Taken together, Ezh2 is essential for the faithful reprogramming of fetal HSPCs into adult 
type in BM.

Another mechanism by which let-7 microRNAs are coordinately repressed is upregulation of competing endogenous RNAs (ceRNAs) for let-7 microRNAs, such as HMGA2 (35). HMGA2 RNA has multiple let-7 binding sites and act as a molecular sponge for let-7 microRNA. When it is overexpressed, it competes for let-7 occupancy with other let-7 targets and causes the upregulation of those targets. As mentioned above, many FL-specific let-7 target genes were de-repressed despite the relatively mild reduction in let-7 levels in Ezh2-deficient HSPCs, and this trend was further enhanced in MDS and MDS/MPN mice. In addition, even the let-7 target genes not regulated by Ezh2, including Arid $3 a$ and Bcl2l1/Bclx (Fig. 7B), became activated in the absence of Ezh2 in BM HSPCs. These findings suggest that let-7 target RNAs that are de-repressed in the absence of Ezh2, including Hmga2, can act as ceRNAs to reinforce de-repression of oncofetal let-7 target genes. In this regard, de-repression of any of the let-7 targets could promote activation of oncofetal let-7 targets (Fig. 8). This ceRNA-like effect could partly contribute to the progressive upregulation of FL-specific let-7 target genes, such as Arid3a and Bcl2ll/Bclx, during the progression of MDS and MDS/MPN post-deletion of Ezh2 (Fig. 7B). Taken together, Ezh2 loss in BM HSPCs could result in activation of let-7 target genes via both transcriptional and post-transcriptional dysregulation of let-7 target genes: transcriptional activation of let-7 target genes via reduced $\mathrm{H} 3 \mathrm{~K} 27 \mathrm{me} 3$ at their promoters, downregulation of let-7 microRNAs by de-repressed Lin28b, and the molecular sponge/ceRNA-like effects of let-7 target RNAs against let-7 microRNAs (Fig. 8).

Reactivation of a fetal gene signature is often associated with development of tumors as is the case with overexpression of Sox17 in HPCs (10). EZH2 functions as oncogenes as well as tumor suppressor genes depending on the tumor types. Inactivating EZH2 mutations are observed in hematological malignancies such as MDS and MDS/MPN and Ezh2 loss 
facilitates development of MDS and MDS/MPN in mice $(20,22,36)$. Activation of a fetal gene signature, particularly oncofetal let-7 targets such as Hmga2, is thought to be responsible for the enhanced repopulating capacity and an MPN-like character of Ezh2-deficient hematopoietic cells $(9,34,37,38)$. Indeed, multiple members of the let-7 family are often repressed in human cancers, thereby promoting oncogenesis by de-repressing their targets such as $H M G A 2, K-R A S$, and $c-M Y C$. Overexpression of LIN28B is one of the mechanisms by which let-7 microRNAs are coordinately repressed and is associated with poor clinical prognosis $(16,18)$. Wilms tumor, the most common pediatric kidney tumor, is one of such tumors in which overexpression of $L I N 28 B$ has a critical role in its pathogenesis (39). Notably, PcG target genes are largely de-repressed in Wilms tumors and murine Wilms-like kidney tumors induced by the transient expression of reprogramming factors in mice (40). These findings suggest that PcG function is compromised in these tumors. Thus, in line with our observation in hematological malignancies, PcG insufficiencies are also closely associated with deregulation of the LIN28B/Let-7 pathway in human tumors.

Gene silencing is established via several different manners. In contrast to the stable form of gene silencing established by combination of $\mathrm{H} 3 \mathrm{~K} 9 \mathrm{me} 3$ and DNA methylation, PcG-mediated silencing is reversible and usually observed with genes that function in the later stage of differentiation or in response to external signals. It remains unknown why a significant portion of FL-specific genes are repressed by Ezh2, but not by H3K9me3 and DNA methylation, in adult BM HSPCs. However, Ezh2 may prevent activation of these potential oncofetal genes in adulthood by restricting ectopic activation in HSPCs, a major target of transformation. 


\section{Experimental procedures}

Mice and generation of hematopoietic chimeras. Tet2 gene trap mice $\left(\operatorname{Tet} 2^{\mathrm{KD} / \mathrm{KD}}\right)(41)$ and $E z h 2^{f l f l}$ mice (19), which had been backcrossed onto a C57BL/6 (CD45.2) background, were used. For conditional deletion of Ezh2, the mice were crossed with Rosa::Cre-ERT mice (TaconicArtemis GmbH). To generate hematopoietic chimeras, we intravenously transplanted E14.5 fetal liver cells from CD45.2 mutant mice (test cells) into 8-week-old CD45.1 recipients irradiated at a dose of 8.5-9.5 Gy. After 1 month, the recipient mice were injected with $100 \mu \mathrm{l}$ of tamoxifen dissolved in corn oil at a concentration of $10 \mathrm{mg} / \mathrm{ml}$ intraperitoneally for 5 consecutive days to induce Cre activity. All experiments using the mice were performed in accordance with our institutional guidelines for the use of laboratory animals and approved by the Review Board for animal experiments of Chiba University (approval ID: 27-213).

Purification of cells and flow cytometry. Monoclonal antibodies (mAbs) recognizing the following antigens were used in flow cytometry and cell sorting: CD45.2 (104), CD45.1 (A20), Gr-1 (RB6-8C5), CD11b/Mac-1 (M1/70), Ter-119 (Ter-119), CD127/Il-7R $\alpha$ (A7R34), B220 (RA3-6B2), CD4 (L3T4), CD8a (53-6.7), CD117/c-Kit (2B8), Sca-1 (D7) and CD16/32/FcyRII-III (93), CD19 (6D5), CD5 (53-7.3), CD1d (1B1), CD23 (B3B4). CD3 (145-2C11) and TCR $\gamma \delta$ (GL3). Fetal liver or BM cells were triturated and passed through $40 \mu \mathrm{m}$ nylon mesh, and then mononuclear cells were isolated on Ficoll-Paque ${ }^{\mathrm{TM}}$ PLUS (GE Healthcare). For magnetic cell sorting, cells were incubated with magnetic beads conjugated with anti-CD117 antibodies (Miltenyi Biotec,) and passed through MACS separation columns. For antibody staining, cells were first incubated in $4^{\circ} \mathrm{C}$ for $30 \mathrm{~min}$ with a mixture of biotin-conjugated mAbs against lineage markers including Gr-1, Mac-1, 
Ter-119, B220, IL-7R $\alpha$, CD4, and CD8 $\alpha$ (Mac-1 was removed from the mixture for fetal liver cells). Next, cells were incubated in $4^{\circ} \mathrm{C}$ for 90 minutes with c-Kit, Sca-1, CD34 and Fc $\gamma$ R. Dead cells were excluded by staining with propidium iodide (Sigma-Aldrich) and flow cytometric experiments were performed on a FACSAria II (BD Biosciences). LSK (lineage marker ${ }^{-} \mathrm{c}-\mathrm{Kit}^{+} \mathrm{Sca}-1^{+}$) and GMP (lineage marker ${ }^{-} \mathrm{K}-\mathrm{Kit}^{+} \mathrm{Sca}-1^{-} \mathrm{CD} 34^{+} \mathrm{Fc} \gamma \mathrm{R}^{+}$) fractions were defined as described previously. The data was analyzed using a FlowJo software (TreeStar).

Microarray analysis. A one-color microarray-based gene expression analysis system (Agilent Technologies) containing 39,429 clones was used (SurePrint G3 Mouse GE 8×60K array; Agilent Technologies) according to the manufacturer's instructions. Total RNA was extracted from $5 \times 10^{4}$ LSK cells or GMPs. A total of $10 \mathrm{ng}$ of total RNA was mixed with spike-in controls using a One Color Spike Mix kit (Agilent Technologies), amplified and labeled with Cyanine 3 using a Quick Amp Labeling kit (Agilent Technologies) according to the manufacturer's instructions, which generated single-color labeled cRNA. A total of $1,650 \mathrm{ng}$ of the labeled cRNA was used for each hybridization. The process of hybridization and washing was performed using a Hi-RPM Gene Expression Hybridization kit (Large; Agilent Technologies) and a Gene Expression Wash Pack (Agilent Technologies), respectively. A DNA microarray scanner (Agilent Technologies) was used for array scanning.

ChIP assay and ChIP-sequencing: LSK cells and GMPs LK were cross-linked with 0.5\% formaldehyde for $5 \mathrm{~min}$ at room temperature, washed twice with PBS, lysed with RIPA buffer (10 mM Tris-HCl pH 8.0, $150 \mathrm{mM} \mathrm{NaCl,} 1$ mM EDTA, 1\% Triton X-100, 0.1\% SDS, $0.1 \%$ sodium deoxycholate [DOC], and protease inhibitor cocktail) and sonicated for $10 \mathrm{sec}$ 
x 15 times (110sec on ice) using a Microson XL2000 Ultrasonic cell disruptor (Misonix). After centrifugation, the soluble chromatin fraction was recovered, precleared for 1 hour at $4^{\circ} \mathrm{C}$ with a 1:1 mixture of Protein A- and G-conjugated Dynabeads (Invitrogen) and then incubated overnight at $4^{\circ} \mathrm{C}$. Chromatin was immunoprecipitated overnight at $4^{\circ} \mathrm{C}$ with Dynabeads conjugated with an anti-H3K27me3 (07-449; Millipore). The immunoprecipitates were rinsed once with RIPA buffer and washed by rotating for 5 minutes four times in RIPA buffer, twice in high salt RIPA buffer $(500 \mathrm{mM} \mathrm{NaCl})$, twice in LiCl buffer (10 mM Tris-HCl pH 8.0, $250 \mathrm{mM} \mathrm{LiCl,} 1 \mathrm{mM}$ EDTA, 0.5\% NP-40, and 0.5\% DOC), and rinsed twice with TE buffer (10 mM Tris-HCl $\mathrm{pH} 8.0$, and $1 \mathrm{mM}$ EDTA). Bound chromatin and input DNA were placed in elution buffer $(10 \mathrm{mM}$ Tris- $\mathrm{HCl}, \mathrm{pH} 8.0,5 \mathrm{mM}$ EDTA, $300 \mathrm{mM} \mathrm{NaCl}$, and $0.5 \% \mathrm{SDS}$ ) and treated sequentially with $5 \mu \mathrm{lof} 1 \mathrm{mg} / \mathrm{ml}$ RNase A (Sigma-Aldrich) for 30 minutes at $37^{\circ} \mathrm{C}, 1 \mu \mathrm{l}$ of $20 \mathrm{mg} / \mathrm{ml}$ proteinase $\mathrm{K}$ (Roche) for 1 hour at $55^{\circ} \mathrm{C}$ and 6 hours at $65^{\circ} \mathrm{C}$ to reverse formaldehyde crosslinking. Eluted DNA was purified with a QIAquick PCR purification kit (QIAGEN). For ChIP assay, quantitative PCR was performed with an ABI StepOnePlus Thermal Cycler using SYBR Premix Ex Taq II (Takara Bio). The primer sequences are listed in Supplemental Experimental Procedures.

For ChIP-Sequencing, $1 \mathrm{ng}$ immunoprecipitated DNA and input samples were prepared using TruSeq ChIP Sample Prep Kit (Illumina) or NEBNext Ultra DNA Library Prep Kit for Illumina (NEB). Adapter-ligated DNA was amplified by 16 to 18 cycles of PCR and DNA libraries were quantified using a high sensitivity Chip on Bioanalyzer (Agilent) and sequenced using Hiseq2500 or Hiseq1500 (Illumina), according to the manufacturer's instructions. 36 to 50 cycles of the sequencing reactions were performed and sequences were aligned to the mouse genome sequences ( $\mathrm{mm} 9$ from University of California, Santa Cruz Genome Browser; http://genome.ucsc.edu/) using the Eland program. Sequences allowing no more than two mismatches per sequence were used for the analysis. 
Sequencing data analysis: The ChIP-Seq signal was quantified as total number of reads per million. To evaluate the histone modification mark of each gene, normalized tag numbers in the region from $2 \mathrm{~kb}$ upstream to $2 \mathrm{~kb}$ downstream of the TSS were counted and divided by tag number of corresponding input. To visualize with Integrative Genomics Viewer (IGV) genome browser, the RPKM (reads per kilobase of transcript per million mapped reads) values of the sequenced reads were calculated every 5,000-base-pair bin with a shifting size of 500 base pairs using bedtools. Then, the read numbers of the immunoprecipitated samples were normalized by subtracting the RPKM values of the input samples in each bin and converted to a bigwig file using wigToBigWig tool. The super-computing resource was provided by Human Genome Center, the Institute of Medical Science, the University of Tokyo (http://sc.hgc.jp/shirokane.html).

Quantitative RT-PCR. Total RNA was isolated using TRIZOL LS solution (Invitrogen) and reverse transcribed using a ThermoScript RT-PCR system (Invitrogen) with an oligo-dT primer. Real-time quantitative RT-PCR (qRT-PCR) was performed with an ABI StepOnePlus Thermal Cycler (Applied Biosystems) using SYBR Premix Ex TaqII (TaKaRa) and primers listed below.

Primer sequences for quantitative RT-PCR are as follows: (forward/reverse primer) Lin28b; 5'-GAGTCCAGGATGATTCCAAGA-3'/5'-TGCTCTGACAGTAATGGCACTT-3' Igf2bp3; 5'-AAACAGCTTTCTCGCTTTGC-3'/5'-TCCGCACTTTAGCATCTGGT-3' Hmga2; 5'-AAGGCAGCAAAAACAAGAGC-3'/5'-GCCGTTTTTCTCCAATGGT-3' Actb; 5'-TCAACACCCCAGCCATGTA-3'/5'-GTGGTACGACCAGAGGCATAC-3'

Gene set enrichment analysis (GSEA). We performed GSEA as described (42). Gene sets 
used are listed in Supplemental Table E4.

Accession numbers. Microarray and ChIP-sequence data obtained in this study were deposited in Gene Expression Omnibus (GEO) (accession number GSE69760) and DNA Data Bank of Japan (DDBJ) (DDBJ, accession number DRA003685), respectively. The microarray and ChIP-sequence data in our previous work (Muto et al., 2013) were retrieved from GEO (accession number GSE42666) and DDBJ (accession number DRA000858), respectively.

Statistical analysis. Unpaired two-tailed Student's t-test was used to assess statistical significance. Statistical tests were carried out using Graph Pad Prism version 5. 


\section{Acknowledgements}

We thank Haruhiko Koseki for Ezh2 mutant mice. This work was supported in part by Grants-in-aid for Scientific Research (\#15H02544) and Scientific Research on Innovative Areas "Stem Cell Aging and Disease" (\#25115002) and "Genome Science” (\#221S0002) from MEXT, Japan, Grant-in-aid for Core Research for Evolutional Science and Technology (CREST) from the Japan Science and Technology Corporation (JST), and grants from the Uehara Memorial Foundation and the Takeda Science Foundation.

\section{Competing financial interests}

The authors declare no competing financial interests. 


\section{REFERENCES}

1. Dzierzak, E., and N.A. Speck. 2008. Of lineage and legacy: the development of mammalian hematopoietic stem cells. Nat Immunol 9:129-136.

2. Ciau-Uitz, A., R. Monteiro, A. Kirmizitas, and R. Patient. 2014. Developmental hematopoiesis: ontogeny, genetic programming and conservation. Exp Hematol 42:669-683.

3. Babovic, S., and C.J. Eaves. 2014. Hierarchical organization of fetal and adult hematopoietic stem cells. Exp Cell Res 329:185-191.

4. Bowie, M.B., K.D. McKnight, D.G. Kent, L. McCaffrey, P.A. Hoodless, and C.J. Eaves. 2006. Hematopoietic stem cells proliferate until after birth and show a reversible phase-specific engraftment defect. J Clin Invest 116:2808-2816.

5. Bowie, M.B., D.G. Kent, B. Dykstra, K.D. McKnight, L. McCaffrey, P.A. Hoodless, and C.J. Eaves. 2007. Identification of a new intrinsically timed developmental checkpoint that reprograms key hematopoietic stem cell properties. Proc Natl Acad Sci USA 104:5878-5882.

6. Harrison, D.E., R.K. Zhong, C.T. Jordan, I.R. Lemischka, and C.M. Astle. 1997. Relative to adult marrow, fetal liver repopulates nearly five times more effectively long-term than short-term. Exp Hematol 25:293-297.

7. McKinney-Freeman, S., P. Cahan, H. Li, S.A. Lacadie, H.T. Huang, M. Curran, S. Loewer, O. Naveiras, K.L. Kathrein, M. Konantz, E.M. Langdon, C. Lengerke, L.I. Zon, J.J. Collins, and G.Q. Daley. 2012. The transcriptional landscape of hematopoietic stem cell ontogeny. Cell Stem Cell 11:701-714.

8. Phillips, R.L., R.E. Ernst, B. Brunk, N. Ivanova, M.A. Mahan, J.K. Deanehan, K.A. Moore, G.C. Overton, and I.R. Lemischka. 2000. The genetic program of hematopoietic stem cells. Science 288:1635-1640. 
9. Copley, M.R., S. Babovic, C. Benz, D.J. Knapp, P.A. Beer, D.G. Kent, S. Wohrer, D.Q. Treloar, C. Day, K. Rowe, H. Mader, F. Kuchenbauer, R.K. Humphries, and C.J. Eaves. 2013. The Lin28b/let-7-Hmga2 axis determines the higher self-renewal potential of fetal haematopoietic stem cells. Nat Cell Biol 15:916-925.

10. He, S., I. Kim, M.S. Lim, and S.J. Morrison. 2011. Sox17 expression confers self-renewal potential and fetal stem cell characteristics upon adult hematopoietic progenitors. Genes Dev 25:1613-1627.

11. Kim, I., T.L. Saunders, and S.J. Morrison. 2007. Sox17 dependence distinguishes the transcriptional regulation of fetal from adult hematopoietic stem cells. Cell $130: 470-483$

12. Park, I.K., D. Qian, M. Kiel, M.W. Becker, M. Pihalja, I.L. Weissman, S.J. Morrison, and M.F. Clarke. 2003. Bmi-1 is required for maintenance of adult self-renewing haematopoietic stem cells. Nature 423:302-305.

13. Iwama, A., H. Oguro, M. Negishi, Y. Kato, Y. Morita, H. Tsukui, H. Ema, T. Kamijo, Y. Katoh-Fukui, H. Koseki, M. van Lohuizen, and H. Nakauchi. 2004. Enhanced self-renewal of hematopoietic stem cells mediated by the polycomb gene product Bmi-1. Immunity 21:843-851.

14. Ye, M., H. Zhang, G. Amabile, H. Yang, P.B. Staber, P. Zhang, E. Levantini, M. Alberich-Jorda, J. Zhang, A. Kawasaki, and D.G. Tenen. 2013. C/EBPa controls acquisition and maintenance of adult haematopoietic stem cell quiescence. Nat Cell Biol $15: 385-394$

15. Nishino, J., I. Kim, K. Chada, and S.J. Morrison. 2008. Hmga2 promotes neural stem cell self-renewal in young but not old mice by reducing p16Ink4a and p19Arf Expression. Cell 135:227-239.

16. Viswanathan, S.R., J.T. Powers, W. Einhorn, Y. Hoshida, T.L. Ng, S. Toffanin, M. 
O'Sullivan, J. Lu, L.A. Phillips, V.L. Lockhart, S.P. Shah, P.S. Tanwar, C.H. Mermel, R. Beroukhim, M. Azam, J. Teixeira, M. Meyerson, T.P. Hughes, J.M. Llovet, J. Radich, C.G. Mullighan, T.R. Golub, P.H. Sorensen, and G.Q. Daley. 2009. Lin28 promotes transformation and is associated with advanced human malignancies. Nat Genet $41: 843-848$

17. Viswanathan, S.R., and G.Q. Daley. 2010. Lin28: A microRNA regulator with a macro role. Cell 140:445-449.

18. Nguyen, L.H., D.A. Robinton, M.T. Seligson, L. Wu, L. Li, D. Rakheja, S.A. Comerford, S. Ramezani, X. Sun, M.S. Parikh, E.H. Yang, J.T. Powers, G. Shinoda, S.P. Shah, R.E. Hammer, G.Q. Daley, and H. Zhu. 2014. Lin28b is sufficient to drive liver cancer and necessary for its maintenance in murine models. Cancer Cell 26:248-261.

19. Mochizuki-Kashio, M., Y. Mishima, S. Miyagi, M. Negishi, A. Saraya, T. Konuma, J. Shinga, H. Koseki, and A. Iwama. 2011. Dependency on the polycomb gene Ezh2 distinguishes fetal from adult hematopoietic stem cells. Blood 118:6553-6561.

20. Muto, T., G. Sashida, M. Oshima, G.R. Wendt, M. Mochizuki-Kashio, Y. Nagata, M. Sanada, S. Miyagi, A. Saraya, A. Kamio, G. Nagae, C. Nakaseko, K. Yokote, K. Shimoda, H. Koseki, Y. Suzuki, S. Sugano, H. Aburatani, S. Ogawa, and A. Iwama. 2013. Concurrent loss of Ezh2 and Tet2 cooperates in the pathogenesis of myelodysplastic disorders. J Exp Med 210:2627-2639.

21. Ernst, T., A.J. Chase, J. Score, C.E. Hidalgo-Curtis, C. Bryant, A.V. Jones, K. Waghorn, K. Zoi, F.M. Ross, A. Reiter, A. Hochhaus, H.G. Drexler, A. Duncombe, F. Cervantes, D. Oscier, J. Boultwood, F.H. Grand, and N.C. Cross. 2010. Inactivating mutations of the histone methyltransferase gene EZH2 in myeloid disorders. Nat Genet 42:722-726.

22. Shih, A.H., O. Abdel-Wahab, J.P. Patel, and R.L. Levine. 2012. The role of mutations in epigenetic regulators in myeloid malignancies. Nat Rev Cancer 12:599-612. 
23. Oshima, M., and A. Iwama. 2014. Epigenetics of hematopoietic stem cell aging and disease. Int J Hematol 100:326-334.

24. Oguro, H., J. Yuan, H. Ichikawa, T. Ikawa, S. Yamazaki, H. Kawamoto, H. Nakauchi, and A. Iwama. 2010. Poised lineage specification in multipotential hematopoietic stem and progenitor cells by the polycomb protein Bmi1. Cell Stem Cell 6:279-286.

25. Hidalgo, I., A. Herrera-Merchan, J.M. Ligos, L. Carramolino, J. Nunez, F. Martinez, O. Dominguez, M. Torres, and S. Gonzalez. 2012. Ezh1 is required for hematopoietic stem cell maintenance and prevents senescence-like cell cycle arrest. Cell Stem Cell 11:649-662.

26. Xie, H., J. Xu, J.H. Hsu, M. Nguyen, Y. Fujiwara, C. Peng, and S.H. Orkin. 2014. Polycomb repressive complex 2 regulates normal hematopoietic stem cell function in a developmental-stage-specific manner. Cell Stem Cell 14:68-80.

27. Yuan, J., C.K. Nguyen, X. Liu, C. Kanellopoulou, and S.A. Muljo. 2012. Lin28b reprograms adult bone marrow hematopoietic progenitors to mediate fetal-like lymphopoiesis. Science 335:1195-1200.

28. Zhou, Y., Y.S. Li, S.R. Bandi, L. Tang, S.A. Shinton, K. Hayakawa, and R.R. Hardy. 2015. Lin28b promotes fetal B lymphopoiesis through the transcription factor Arid3a. $J$ Exp Med 212:569-580.

29. Baumgarth, N. 2011. The double life of a B-1 cell: self-reactivity selects for protective effector functions. Nat Rev Immunol 11:34-46.

30. Kikuchi, K., and M. Kondo. 2006. Developmental switch of mouse hematopoietic stem cells from fetal to adult type occurs in bone marrow after birth. Proc Natl Acad Sci US A 103:17852-17857.

31. Yoshimoto, M., E. Montecino-Rodriguez, M.J. Ferkowicz, P. Porayette, W.C. Shelley, S.J. Conway, K. Dorshkind, and M.C. Yoder. 2011. Embryonic day 9 yolk sac and 
intra-embryonic hemogenic endothelium independently generate a B-1 and marginal zone progenitor lacking B-2 potential. Proc Natl Acad Sci U S A 108:1468-1473.

32. Pellagatti, A., M. Cazzola, A. Giagounidis, J. Perry, L. Malcovati, M.G. Della Porta, M. Jadersten, S. Killick, A. Verma, C.J. Norbury, E. Hellstrom-Lindberg, J.S. Wainscoat, and J. Boultwood. 2010. Deregulated gene expression pathways in myelodysplastic syndrome hematopoietic stem cells. Leukemia 24:756-764.

33. Guglielmelli, P., R. Zini, C. Bogani, S. Salati, A. Pancrazzi, E. Bianchi, F. Mannelli, S. Ferrari, M.C. Le Bousse-Kerdiles, A. Bosi, G. Barosi, A.R. Migliaccio, R. Manfredini, and A.M. Vannucchi. 2007. Molecular profiling of CD34+ cells in idiopathic myelofibrosis identifies a set of disease-associated genes and reveals the clinical significance of Wilms' tumor gene 1 (WT1). Stem Cells 25:165-173.

34. Harada-Shirado, K., K. Ikeda, K. Ogawa, H. Ohkawara, H. Kimura, T. Kai, H. Noji, S. Morishita, N. Komatsu, and Y. Takeishi. 2015. Dysregulation of the MIRLET7/HMGA2 axis with methylation of the CDKN2A promoter in myeloproliferative neoplasms. Br J Haematol 168:338-349.

35. Kumar, M.S., E. Armenteros-Monterroso, P. East, P. Chakravorty, N. Matthews, M.M. Winslow, and J. Downward. 2014. HMGA2 functions as a competing endogenous RNA to promote lung cancer progression. Nature 505:212-217.

36. Sashida, G., H. Harada, H. Matsui, M. Oshima, M. Yui, Y. Harada, S. Tanaka, M. Mochizuki-Kashio, C. Wang, A. Saraya, T. Muto, Y. Hayashi, K. Suzuki, H. Nakajima, T. Inaba, H. Koseki, G. Huang, T. Kitamura, and A. Iwama. 2014. Ezh2 loss promotes development of myelodysplastic syndrome but attenuates its predisposition to leukaemic transformation. Nat Commun 5:4177.

37. Ikeda, K., P.J. Mason, and M. Bessler. 2011. 3'UTR-truncated Hmga2 cDNA causes MPN-like hematopoiesis by conferring a clonal growth advantage at the level of HSC 
in mice. Blood 117:5860-5869.

38. Oguro, H., J. Yuan, S. Tanaka, S. Miyagi, M. Mochizuki-Kashio, H. Ichikawa, S. Yamazaki, H. Koseki, H. Nakauchi, and A. Iwama. 2012. Lethal myelofibrosis induced by Bmil-deficient hematopoietic cells unveils a tumor suppressor function of the polycomb group genes. J Exp Med 209:445-454.

39. Urbach, A., A. Yermalovich, J. Zhang, C.S. Spina, H. Zhu, A.R. Perez-Atayde, R. Shukrun, J. Charlton, N. Sebire, W. Mifsud, B. Dekel, K. Pritchard-Jones, and G.Q. Daley. 2014. Lin28 sustains early renal progenitors and induces Wilms tumor. Genes Dev 28:971-982.

40. Ohnishi, K., K. Semi, T. Yamamoto, M. Shimizu, A. Tanaka, K. Mitsunaga, K. Okita, K. Osafune, Y. Arioka, T. Maeda, H. Soejima, H. Moriwaki, S. Yamanaka, K. Woltjen, and Y. Yamada. 2014. Premature termination of reprogramming in vivo leads to cancer development through altered epigenetic regulation. Cell 156:663-677.

41. Shide, K., T. Kameda, H. Shimoda, T. Yamaji, H. Abe, A. Kamiunten, M. Sekine, T. Hidaka, K. Katayose, Y. Kubuki, S. Yamamoto, T. Miike, H. Iwakiri, S. Hasuike, K. Nagata, K. Marutsuka, A. Iwama, T. Matsuda, A. Kitanaka, and K. Shimoda. 2012. TET2 is essential for survival and hematopoietic stem cell homeostasis. Leukemia 26:2216-2223.

42. Subramanian, A., P. Tamayo, V.K. Mootha, S. Mukherjee, B.L. Ebert, M.A. Gillette, A. Paulovich, S.L. Pomeroy, T.R. Golub, E.S. Lander, and J.P. Mesirov. 2005. Gene set enrichment analysis: a knowledge-based approach for interpreting genome-wide expression profiles. Proc Natl Acad Sci U S A 102:15545-15550. 


\section{Figure legend}

\section{Figure 1. Experimental design.}

We transplanted E14.5 fetal liver cells from Cre-ERT control, Cre-ERT;Ezh2 $2^{f l f l}$ and Cre-ERT;Ezh $2^{f l f l} T e t 2^{K D / K D}$ mice (CD45.2) into lethally irradiated CD45.1 recipient mice and deleted Ezh2 by intraperitoneal injection of tamoxifen at 4 weeks post-transplantation (WT, $E z h 2^{4 / \Delta}$ and $E z h 2^{\Delta / \Delta} T e t 2^{K D / K D}$ mice, respectively). Tet $2^{K D / K D}$ mice denote Tet2 gene trap mice (20). At 3 months post-deletion of Ezh2 (pre-disease stage), LSK cells and GMPs were purified from recipient mice and were subjected to microarray analysis (LSK cells and GMPs) and ChIP-sequencing of H3K27me3 (GMPs). Ezh2 $2^{4 / \Delta} T e t 2^{K D / K D}$ LSK cells purified from recipient mice that developed MDS or MDS/MPN were also subjected to microarray analysis (left panel, published in Ref. 20). In this study, we performed microarray analysis (LSK cells) and ChIP-sequencing of H3K27me3 (LSK cells and GMPs) using E15.5 fetal liver and BM of 8-week-old mice (right panel).

\section{Figure 2. Fetal gene signature is activated in Ezh2-deficient BM HSPCs.}

(A) Scatter diagrams of microarray analysis. LSK HSPCs were isolated from WT and $E z h 2^{4 / \Delta}$ mice at 3 months post-deletion of Ezh2 and from E15.5 fetal liver and BM of 8-week-old mice, then analyzed by microarray-based expression analysis. The signal levels of RefSeq genes in $E z h 2^{\Delta / \Delta}$ LSK cells compared with WT LSK cells (left panel) and in fetal liver LSK cells compared with BM LSK cells (right panel) are plotted. The light gray lines represent the boundaries for 2-fold increase and 2-fold decrease, respectively. Genes upregulated more than 2-fold (or 5-fold for genes which became transcriptionally detectable from undetectable levels) in $E z h 2^{4 / \Delta}$ BM LSK cells ( $E z h 2^{\Delta / \Delta}$ LSK UP genes) compared with WT BM LSK cells (left panel) and fetal liver LSK cells compared with BM LSK cells 
(FL-specific genes) (right panel) are plotted in red. (B) Venn diagram showing the overlap between genes up-regulated ( $\geq 2$ folds) in fetal liver LSK cells compared with BM LSK cells (FL-specific genes) and those in $E z h 2^{4 / \Delta}$ BM LSK cells compared with WT BM LSK cells $\left(E z h 2^{4 / \Delta}\right.$ LSK UP genes) in (A). $p$-value $<1.0 \times 10^{-16}$. (C) A GSEA plot demonstrating a significant positive enrichment of fetal liver-specific genes in $E z h 2^{\Delta / \Delta}$ BM LSK cells compared with WT BM LSK cells. Normalized enrichment score (NES), nominal p-value and false discovery rate (FDR) are indicated. The gene set specific to fetal liver LSK cells defined in (A) was applied to GSEA analysis. (D) A scatter plot showing the correlation of fold expression of RefSeq genes in the fetal liver LSK cells relative to BM LSK cells (FL/BM) and that in $E z h 2^{4 / \Delta}$ BM LSK cells relative to WT BM LSK cells $\left(E z h 2^{4 / 4} / \mathrm{WT}\right)$. The light dotted line represents the boundary for 2-fold increase in the FL/BM values. FL-specific genes defined in (A) are plotted in red. The score of correlation coefficient between the FL/BM and Ezh2 $2^{4 / 4} / \mathrm{WT}$ values defined with Pearson's correlation and the linear regression are shown. (E, F) GSEA plots demonstrating the enrichment trends of $E z h 2^{\Delta / \Delta}$ LSK UP genes $(\mathbf{E})$ and FL-specific genes $(\mathbf{F})$ defined in $(\mathbf{A})$ in $E e d^{\Delta / \Delta} \mathrm{HSCs}, E z h 1^{4 / \Delta}$ HSCs, Bmi $1^{-/}$LSK cells and BM HPCs overexpressing Sox17. NES, nominal p-value and FDR are indicated. (G) $E z h 2^{4 / \Delta}$ BM LSK cells show fetal gene signature. GSEA plots demonstrating a significant positive enrichment of gene expression signatures of developing murine HSPCs retrieved from published database (7) in $E z h 2^{4 / 4}$ BM LSK cells compared with WT cells. NES, nominal p-value and FDR are indicated.

\section{Figure 3. De-repression of let-7 target genes in Ezh2-deficient BM HSPCs}

$(\mathbf{A}, \mathbf{B})$ Quantitative RT-PCR analysis of the expression of Lin28b, Hmga2, Igf $2 b p 3$, let-7b and $l e t-7 c$ in LSK HSPCs and myeloid progenitors (GMP and MEP) from WT E14.5 fetal liver and 8-week-old adult BM (A) and WT and $E z h 2^{4 / \Delta}$ mice at 3 months post-deletion of 
Ezh2 (B). Fold change is indicated above the cell populations compared. $\beta$-actin $(A c t b)$ was used to normalize the amount of input RNA. Data are shown as the mean $\pm \mathrm{SD}$ for triplicate analyses. (C) A scatter plot showing the correlation of fold expression of RefSeq genes in the FL LSK cells relative to BM LSK cells (FL/BM) and that in $E z h 2^{4 / \Delta}$ BM LSK cells relative to WT BM LSK cells $\left(E z h 2^{4 / \Delta} / \mathrm{WT}\right)$. The light dotted line represents boundary for 2-fold increase in the FL/BM values. FL-specific let-7 target genes are plotted in red and the score of the correlation coefficient between the FL/BM and $E z h 2^{\Delta / 4} / \mathrm{WT}$ values (defined with Pearson's correlation) and the linear regression are shown. (D) Box-and-whisker plots showing fold expression levels of indicated genes in $E z h 2^{4 / \Delta}$ BM LSK cells relative to WT BM LSK cells $\left(E z h 2^{4 / \Delta} / \mathrm{WT}\right)$. Boxes represent 25-75 percentile ranges. Vertical lines represent 10-90 percentile ranges. Horizontal bars represent median. (E) GSEA plots demonstrating a significant positive enrichment of FL-specific let-7 target genes in $E z h 2^{4 / \Delta}$ BM LSK cells and GMPs compared with WT cells. NES, nominal p-value and FDR are indicated. Genes highly enriched are indicated at the bottom. (F) GSEA plots demonstrating the enrichment trends of FL-specific let-7 target genes in $E e d^{\Delta / \Delta} \mathrm{HSCs}, \mathrm{Bmil}^{-/-} \mathrm{LSK}$ cells, and BM HPCs overexpressing Sox17. NES, nominal p-value and FDR are indicated. ${ }^{*} p<$ $0.05, * * p<0.01, * * * p<0.001 ;$ ns, not significant.

\section{Figure 4. Active fetal-like lymphopoiesis by Ezh2-deficient adult BM HSPCs}

Lymphopoiesis in recipient mice at 3 months post-deletion of Ezh2 was examined by flow cytometric analysis. Representative flow cytometric profiles of $\mathrm{CD} 45.2^{+} \mathrm{CD} 19^{+}$ donor-derived B cells in the peritoneal cavity (A), CD45. $2^{+} \mathrm{B} 220^{+}$donor-derived B cells in the spleen (B) and CD45.2 $2^{+}$donor-derived thymocytes in the thymus (C) (left panels). $\mathrm{B} 220^{\mathrm{lo}} \mathrm{CD}^{+}$B-1a, B220 ${ }^{\mathrm{lo}} \mathrm{CD}^{-} \mathrm{B}-1 \mathrm{~b}$ and B220 ${ }^{\mathrm{hi}} \mathrm{CD}^{-}$B-2 B cells $(\mathrm{A}), \mathrm{CD}^{-} \mathrm{d}^{+} \mathrm{CD}^{-} 3^{-} \mathrm{MZ}$ and $\mathrm{CD} 1 \mathrm{~d}^{-} \mathrm{CD} 23^{+}$FO B cells $(\mathbf{B})$ and $\mathrm{CD}^{+}{ }^{+} \mathrm{TCR} \gamma \delta^{+} \gamma \delta$ T cells $(\mathbf{C})$ are indicated (left panels) 
and their proportions are shown as scatter diagrams with mean values (bars) $(n=7-8)$ (right panels). ${ }^{*} p<0.05, * * * p<0.001 ; \mathrm{ns}$, not significant.

\section{Figure 5. Ezh2 targets let-7 target genes in adult BM HSPCs}

(A) Inverse correlation between $\mathrm{H} 3 \mathrm{~K} 27 \mathrm{me} 3$ and gene expression levels. Scatter plots showing the correlation of the fold enrichment values of H3K27me3 against the input signals (ChIP/input) from $2.0 \mathrm{~kb}$ upstream to $2.0 \mathrm{~kb}$ downstream of the TSSs of RefSeq genes with the microarray signals in 8-week-old BM LSK cells (left panel) and E15.5 fetal liver LSK cells (right panel). The correlation coefficient and the linear regression are shown. (B) Box-and-whisker plots showing the fold enrichment values of H3K27me3 against the input signals (ChIP/input) (TSS $\pm 2.0 \mathrm{~kb}$ of indicated genes) in FL LSK cells relative to BM LSK cells (FL/BM). Boxes represent 25-75 percentile ranges. Vertical lines represent 10-90 percentile ranges. Horizontal bars represent median. $* * * p<0.001$; ns, not significant. (C) A scatter plots showing the correlation of the fold enrichment values of H3K27me3 against the input signals (ChIP/input) (TSS $\pm 2.0 \mathrm{~kb}$ of RefSeq genes) between FL (E15.5) and BM (8-week-old) LSK cells (left panel). The light horizontal and diagonal lines represent the boundaries for 2-fold enrichment of H3K27me3 in FL LSK cells and 2-fold increase in fold enrichment of H3K27me3 in BM LSK cells compared with FL, respectively (left panel). 503 genes in the yellow gate acquired the H3K27me3 modification ( $\geq 2$-fold increase) in BM LSK cells compared with FL LSK cells, and these genes were defined as BM-specific PRC2 target genes (left panel). A scatter plot showing the correlation of expression of RefSeq genes in the FL LSK cells relative to BM LSK cells (FL/BM) and H3K27me3 levels of RefSeq genes in FL LSK cells relative to adult BM LSK cells (FL/BM) (right panel). The light horizontal and vertical lines represent the boundaries for 2-fold increase in expression in FL LSK cells compared with BM LSK cells and 2-fold reduction in fold enrichment of 
H3K27me3 in fetal liver LSK cells compared with BM LSK cells, respectively. Among 503 BM-specific PRC2 target genes, 81 genes were FL-specific genes showing greater than 2 fold higher expression in FL LSK cells than in BM LSK cells and these genes are indicated in red. The remaining 422 genes were indicated in blue. (D) A scatter plots as in (C) featuring let-7 target genes. Among all let-7 target genes, 44 genes underwent a $\geq 2$-fold increase in the levels of H3K27me3 in adult BM LSK cells compared with FL LSK cells (left panel) and 17 genes were FL-specific let-7 target genes (right panel).

\section{Figure 6. Ezh2 keeps let-7 target genes transcriptionally repressed in adult BM HSPCs}

(A) A scatter plots showing the correlation of the fold enrichment values (ChIP/input) (TSS $\pm 2.0 \mathrm{~kb}$ ) of H3K27me3 against the input signals of RefSeq genes (left panel), FL-specific genes (middle panel) and FL-specific let-7 target genes (right panel) between WT and $E z h 2^{4 / \Delta}$ GMPs from recipient mice at 3 months post-deletion of Ezh2. (B) Box-and-whisker plots showing the fold enrichment (ChIP/input) values of H3K27me3 in $E z h 2^{4 / 4}$ GMPs relative to WT GMPs $\left(E z h 2^{\Delta / 4} / \mathrm{WT}\right)$ are depicted for indicated genes. Boxes represent 25-75 percentile ranges. Vertical lines represent 10-90 percentile ranges. Horizontal bars represent median. $* * p<0.01, * * * p<0.001$. (C) A scatter plot showing the correlation of fold changes in expression and H3K27me3 levels in Ezh2 $2^{\Delta / \Delta}$ GMPs against WT GMPs $\left(E z h 2^{\Delta / \Delta} / \mathrm{WT}\right)$. FL-specific genes (left panel) and FL-specific let-7 target genes (right panel) are plotted in red and the correlation coefficient between changes in expression and H3K27me3 levels as well as the linear regression are shown in red. (D) Visualization of ChIP-sequence data of H3K27me3 levels of representative let-7 target genes in E14.5 FL LSK cells and 8-week-old BM LSK cells (upper panels) and GMPs (middle panels), and GMPs purified from WT and $E z h 2^{\Delta / \Delta}$ mice at 3 months post-deletion of Ezh2 (lower panels) using the Integrative Genomics Viewer (IGV). Schematic diagram of these gene loci 
indicates their genomic structures. Exons and untranslated regions are demarcated by large and small black boxes, respectively.

Figure 7. Escape of let-7 target genes from Ezh2-mediated silencing is associated with progression of hematological malignancies

(A) GSEA plots demonstrating a significant positive enrichment of FL-specific genes (upper panels) and FL-specific let-7 target genes (lower panels) in $E z h 2^{4 / \Delta} \operatorname{Tet} 2^{K D / K D} \mathrm{BM}$ LSK cells from $E z h 2^{\Delta / \Delta} T e t 2^{K D / K D}$ mice at 3 months post-deletion of Ezh2 (pre-disease state) and $E z h 2^{4 / 4} T e t 2^{K D / K D}$ mice that developed MDS and MDS/MPN compared with control WT LSK cells. NES, nominal p-value and FDR are indicated. (B) Graphic presentation of microarray data of selected let-7 target genes in LSK cells from WT, $E z h 2^{4 / \Delta}$ and $E z h 2^{4 / \Delta} T e t 2^{K D / K D}$ mice at 3 months post-deletion of Ezh2, and $E z h 2^{4 / \Delta} T e t 2^{K D / K D}$ mice that developed MDS and MDS/MPN. (C) GSEA plots demonstrating a significant positive enrichment of FL-specific let-7 target genes in $\mathrm{CD} 34^{+}$cells from MDS patients with 7q deletion compared with those from healthy controls. (D) Relative expression of selected FL-specific let-7 target genes in $\mathrm{CD} 4^{+}$cells from healthy controls, MDS patients and MDS patients with $7 \mathrm{q}$ deletion. The data are presented as scatter diagrams with mean values (bars). ${ }^{*} p<0.05, * * p<0.01,{ }^{* * *} p<0.001$; ns, not significant. The data from MDS patients were retrieved from published database (32).

\section{Figure 8. Proposed model for the regulation of let-7 pathway genes by Ezh2.}

In the absence of Ezh2, a cohort of fetal-specific genes, including let-7 target genes, are activated in BM HSPCs, leading to acquisition of fetal phenotypes by BM HSPCs. Many of the fetal-specific let-7 target genes, which are negatively regulated by let-7 microRNAs in a post-transcriptional manner in BM HSPCs, are also transcriptionally repressed by 
Ezh2-mediated H3K27me3 in BM HSPCs. Ezh2 loss in BM HSPCs could induce their transcriptional activation through reduced H3K27me3 levels at their promoters, suppressed let-7 biogenesis by de-repressed Lin28b and the molecular sponge/ceRNA-like effects of activated let-7 target genes against let-7 microRNAs. 


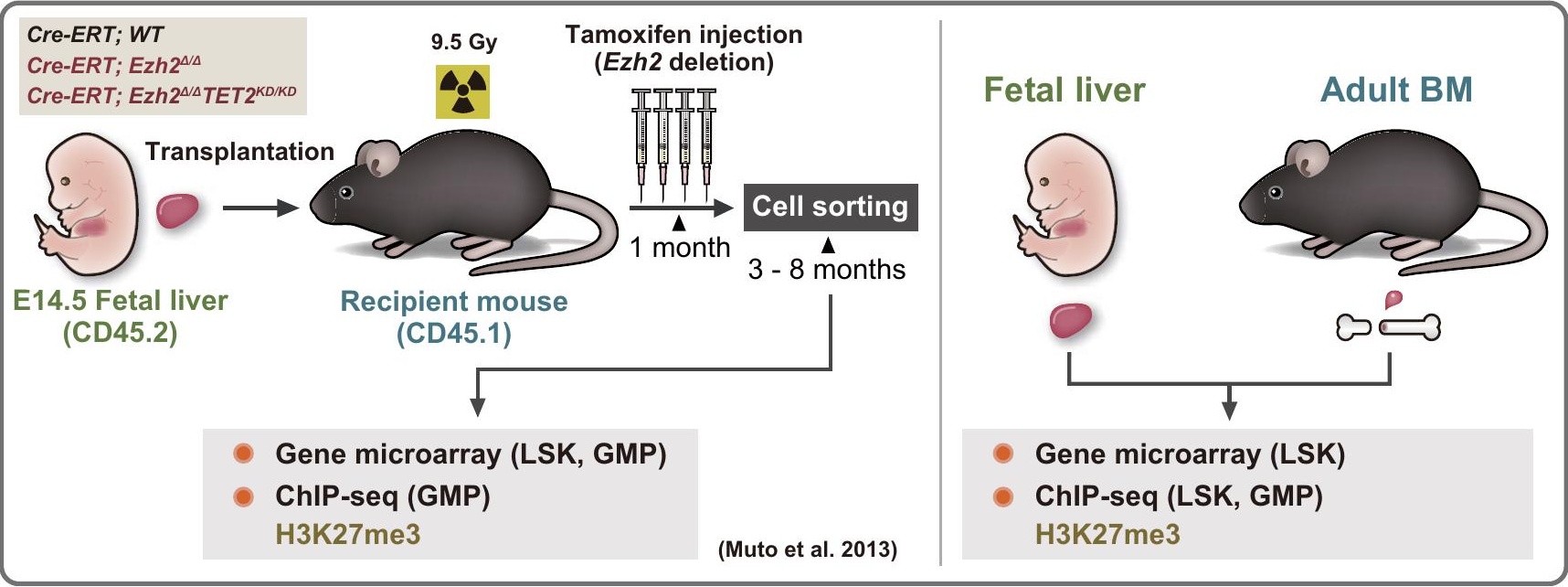


A

Gene expression

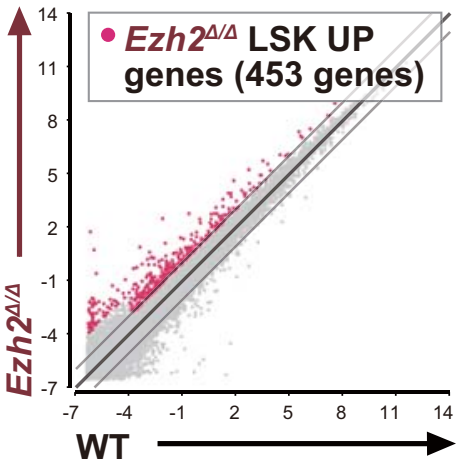

B

FL-specific genes

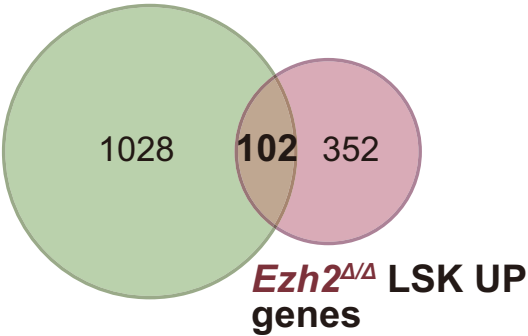

$p$-value $<1.0 \times 10^{-16}$
Gene expression

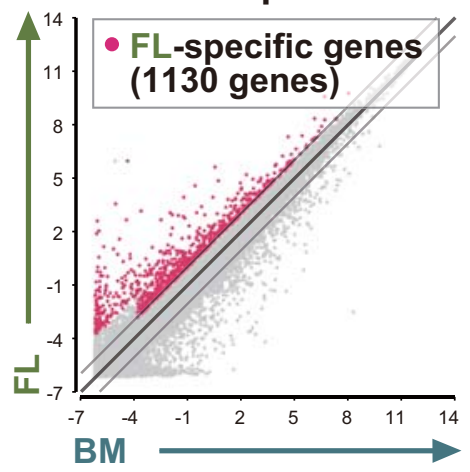

C

FL-specific genes

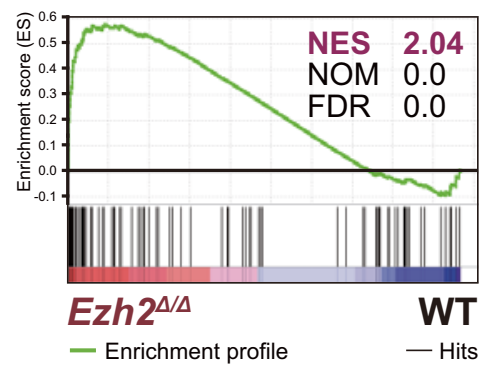

D

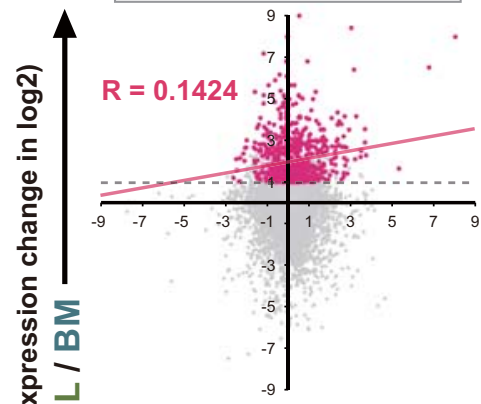

Ezh2 $2^{\Delta / \Delta} / \mathrm{WT}$

(Expression change in $\log 2)$

E

Ezh2 ${ }^{\Delta / \Delta}$ LSK UP genes

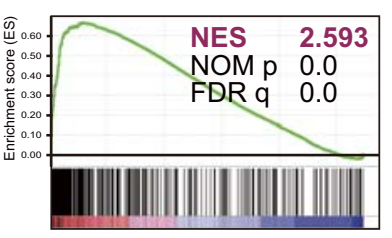

Eed KO

$\mathbf{F}$

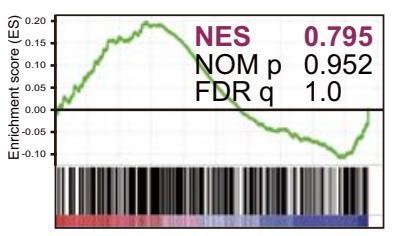

Ezh1 KO

WT

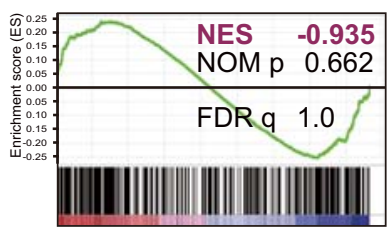

Bmi1 KO

WT

FL-specific genes

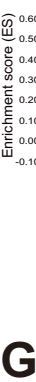

Yolk Sac (CD41+c-Kit+CD34+)

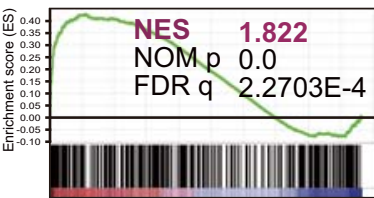

$E z h 2^{\Delta / \Delta}$

WT

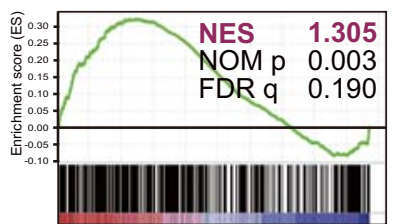

WT

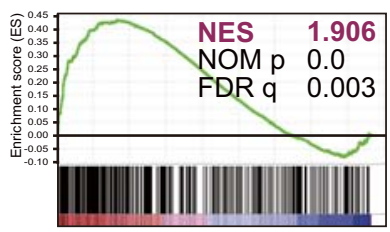

WT

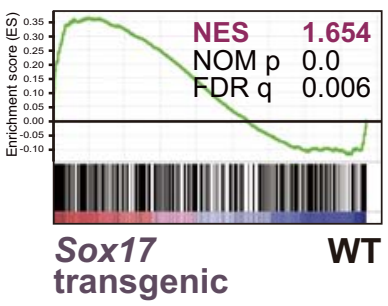

transgenic

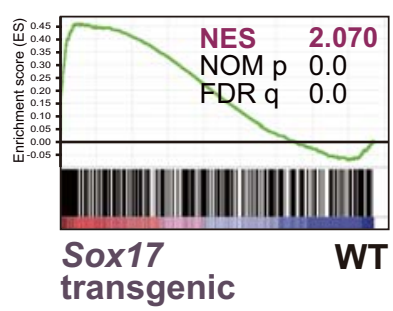

PLACENTA (CD45'c-Kit'CD34MED) AGM (VE-Cad+CD45')

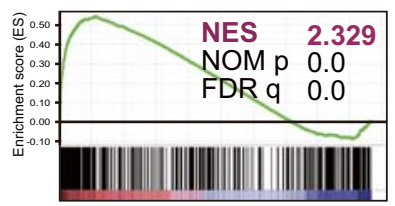

$E z h 2^{\Delta / \Delta}$

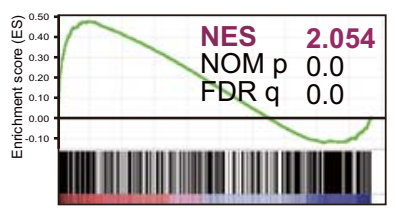

WT
FL E12.5 (VE-CAD+MAC1 ${ }^{\text {LOW }}$ LSK)

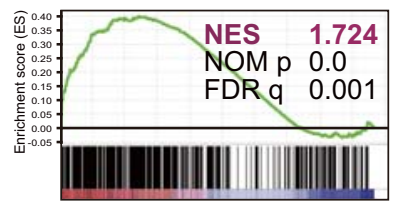


A
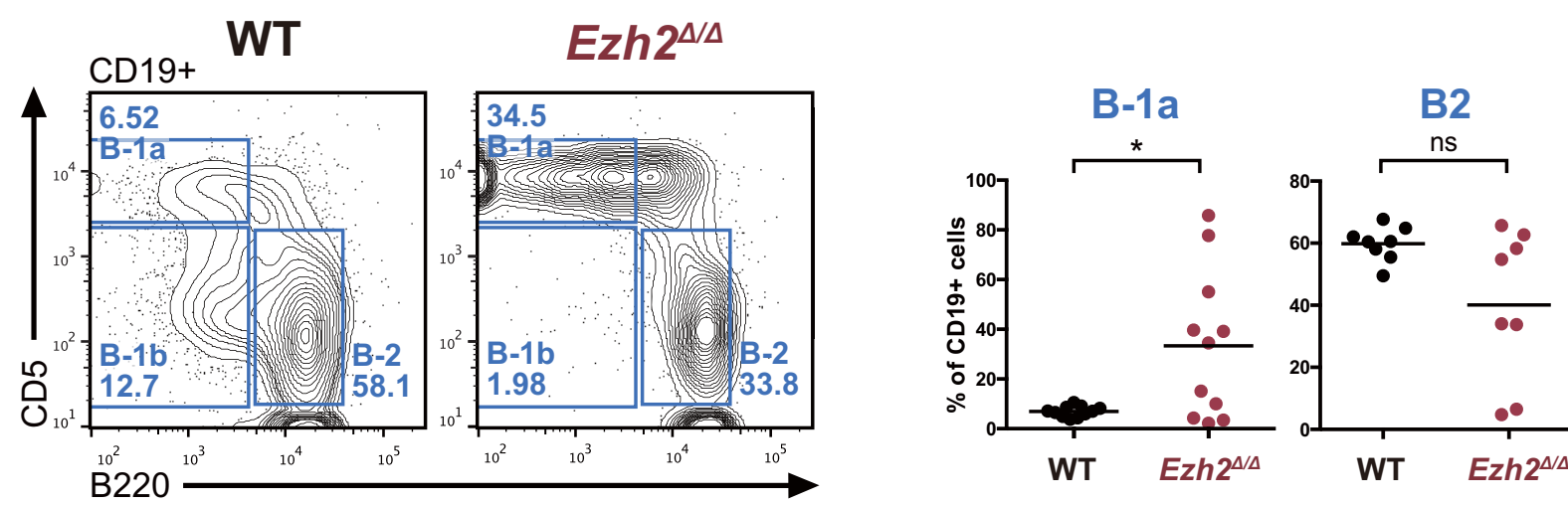

B
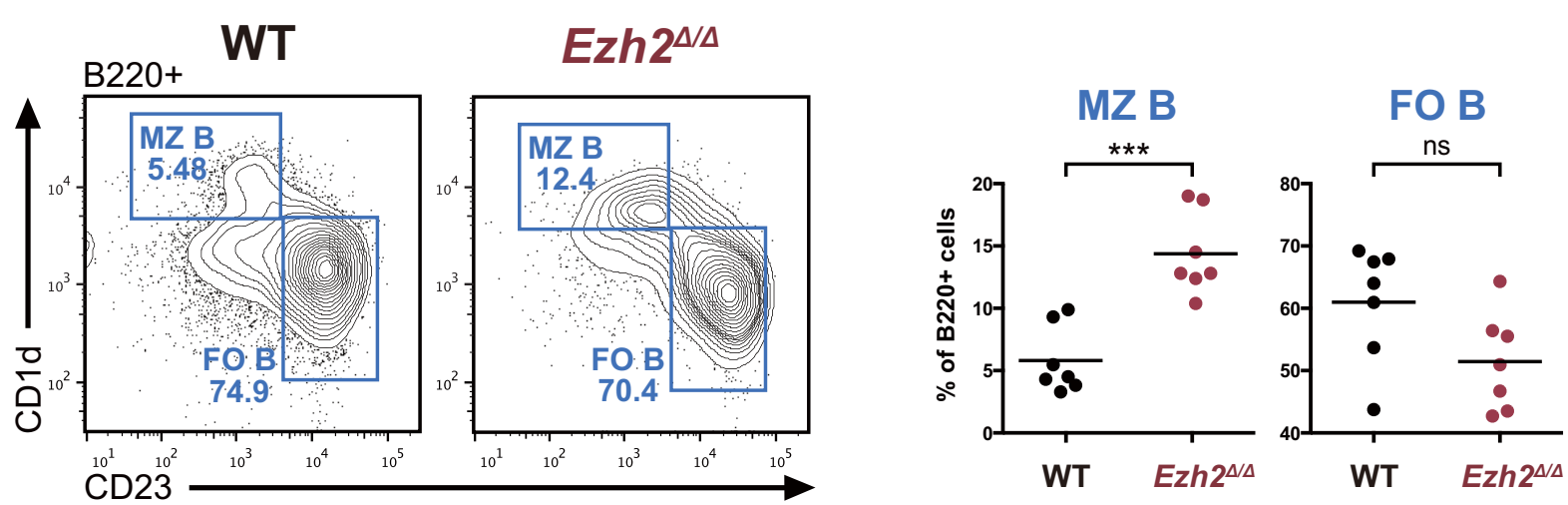

C

WT

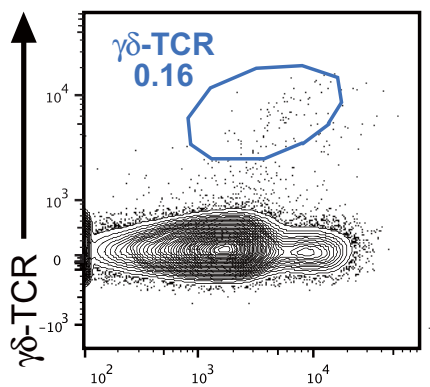

CD3
$\operatorname{Ezh}^{\Delta / \Delta}$

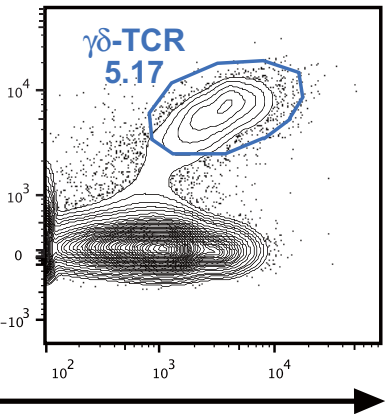

$\gamma \delta$-TCR

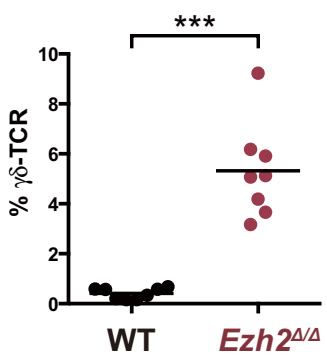


A

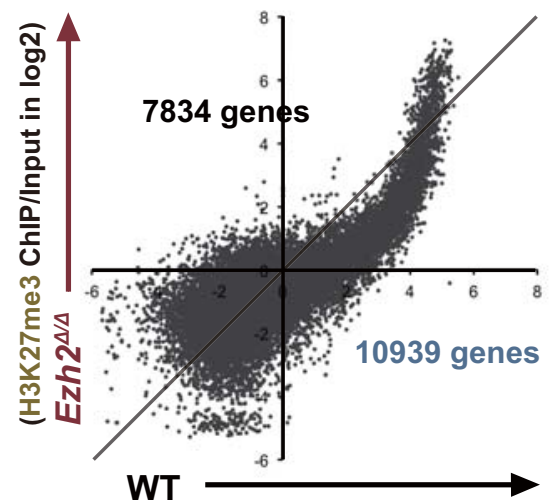

(H3K27me3 ChIP/Input in log2)

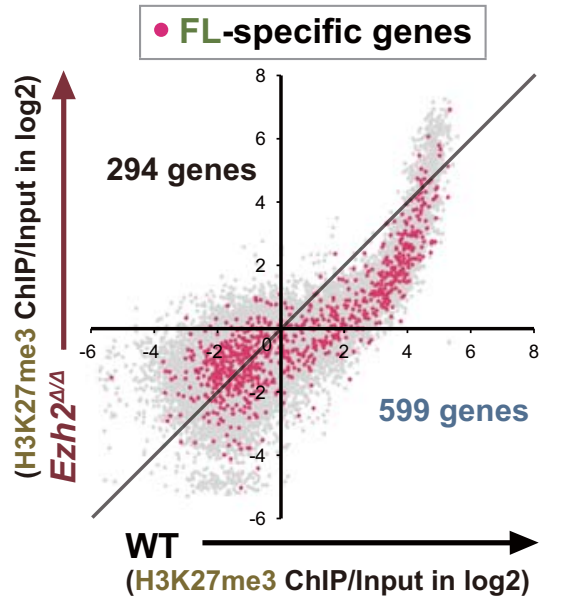

C

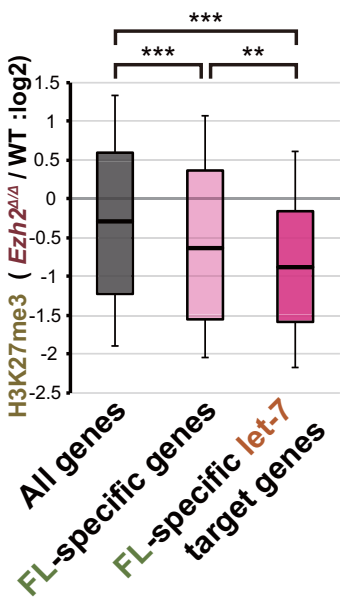

- FL-specific let-7 target genes

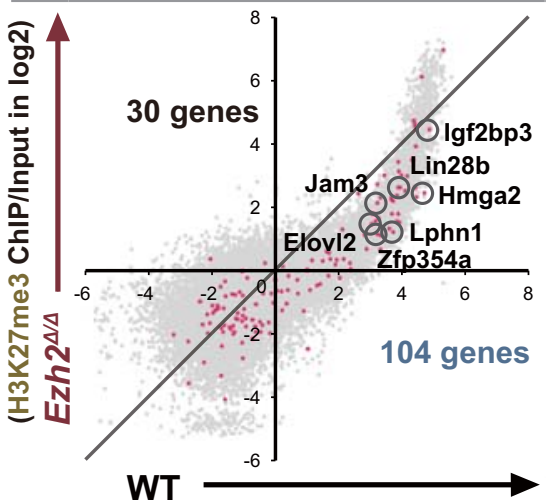

(H3K27me3 ChIP/Input in log2)
- FL-specific let-7 target genes

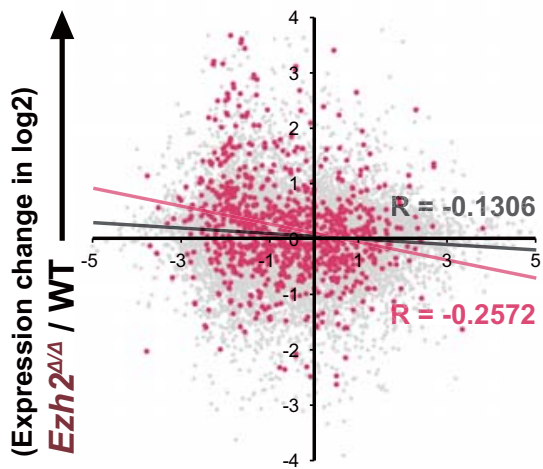

Ezh2 $^{\Delta / \Delta}$ / WT

(H3K27me3 change in log2)

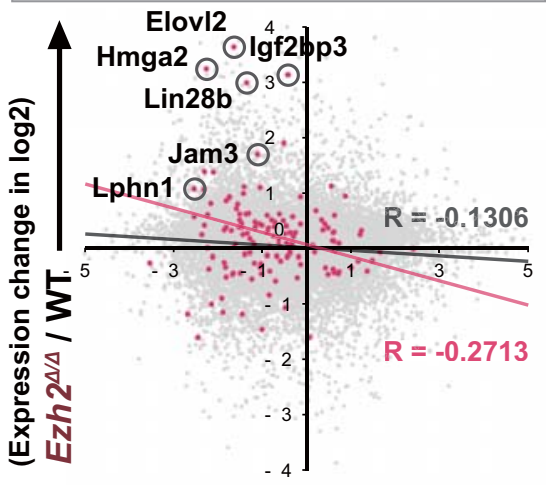

Ezh2 $^{\Delta / \Delta} /$ WT

(H3K27me3 change in log2)

D

LSK

GMP

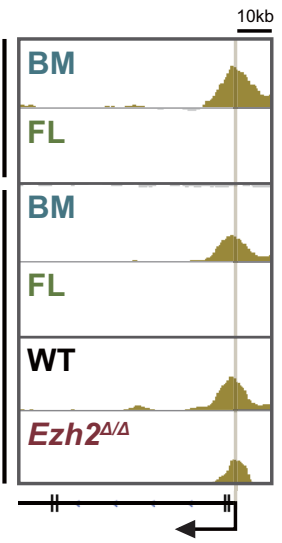

Lin 28b

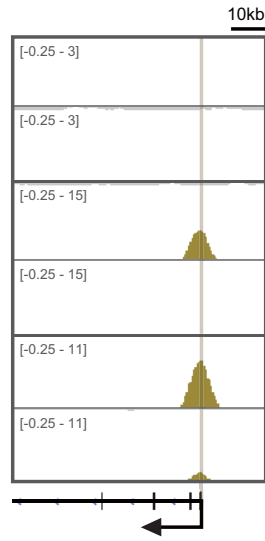

Hmga2

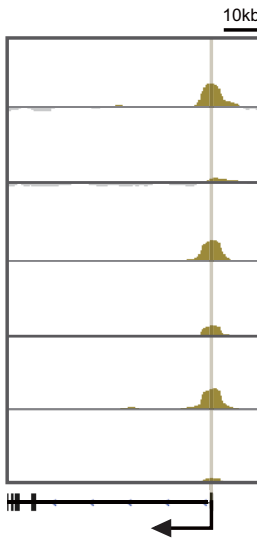

Jam3

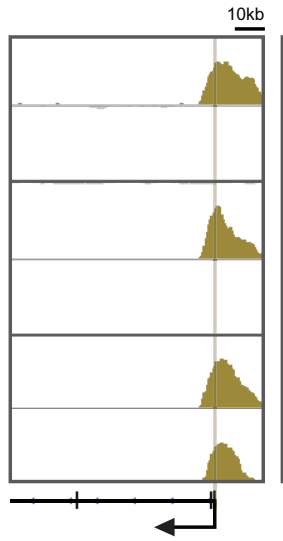

Igf2bp3

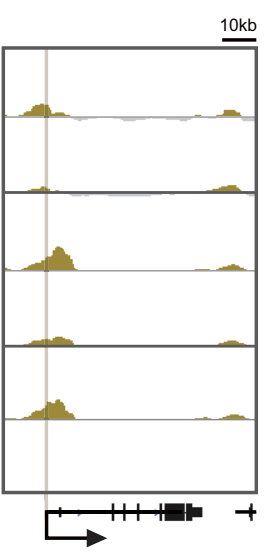

Lphn1

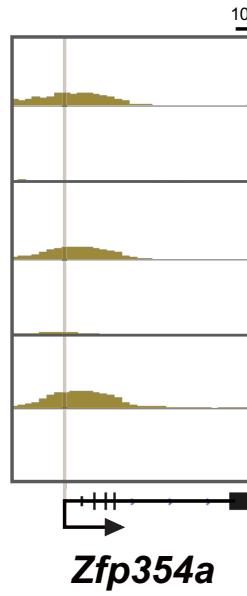




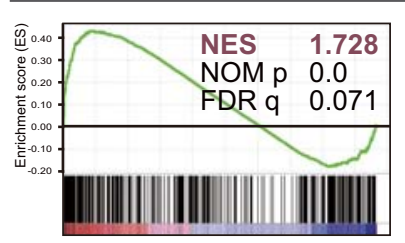

Ezh2 ${ }^{\Delta / \Delta}$ TET2 ${ }^{K D / K D} \quad$ WT

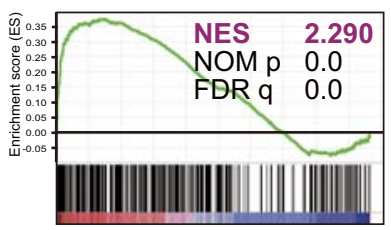

$E_{\text {EZh2 }}{ }^{\Delta / \Delta}$ TET2 ${ }^{K D / K D} \quad$ WT -MDS

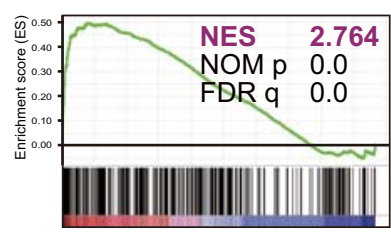

Ezh2 $2^{\Delta / \Delta} T E T 2^{K D / K D} \quad$ WT -MDS/MPN

FL-specific let-7 target genes

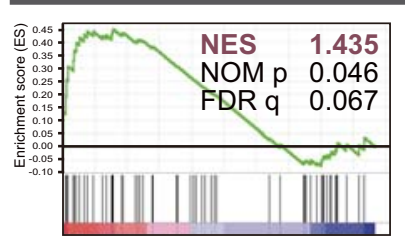

$E_{z h 2^{\Delta / \Delta} T E T 2^{K D / K D} \quad \text { WT }}$

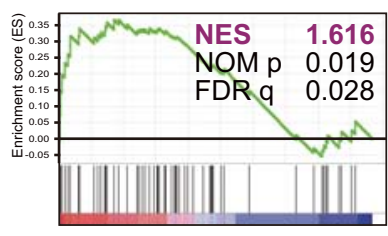

Ezh2 ${ }^{\Delta / \Delta} T E T 2^{K D / K D} \quad$ WT -MDS

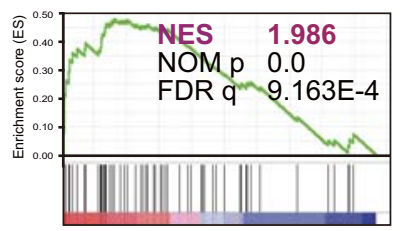

Ezh2 $2^{\Delta / \Delta}$ TET2 ${ }^{K D / K D} \quad$ WT -MDS/MPN
B

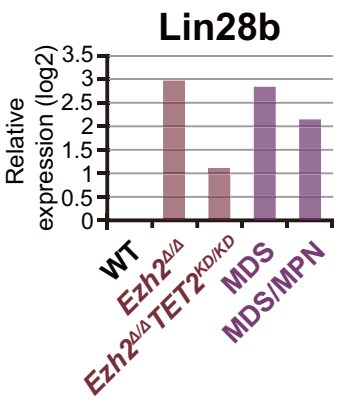

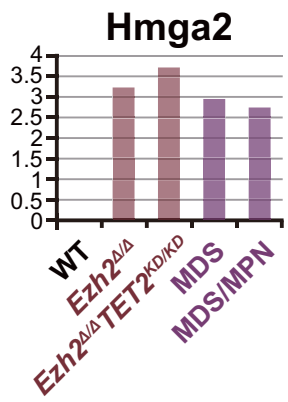

Igf2bp3

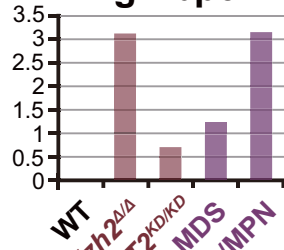

FL-specific let-7 target genes

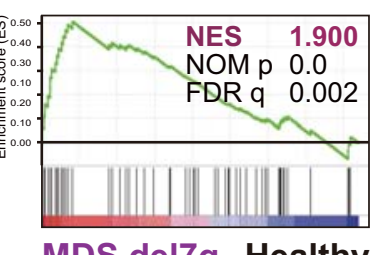

MDS del7q Healthy

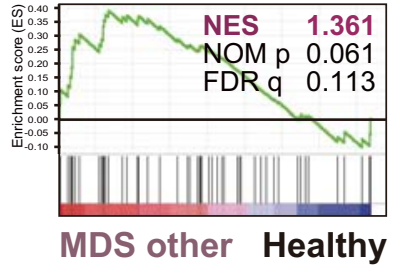

MDS other Healthy
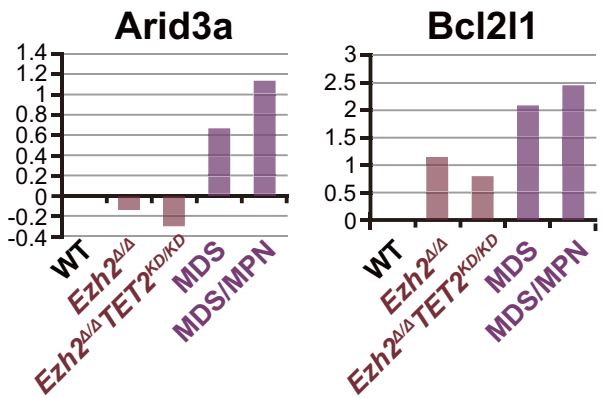
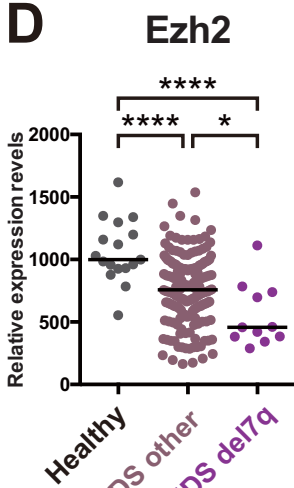

IGF2BP2

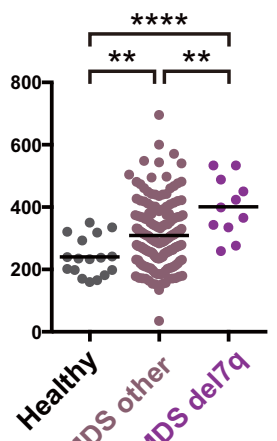

IGF2BP3

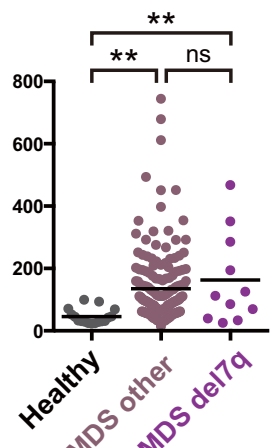

FL-specific genes

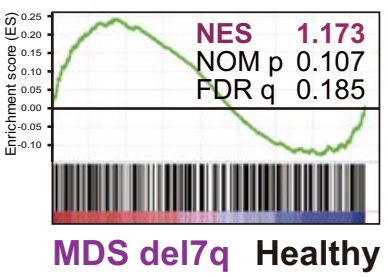

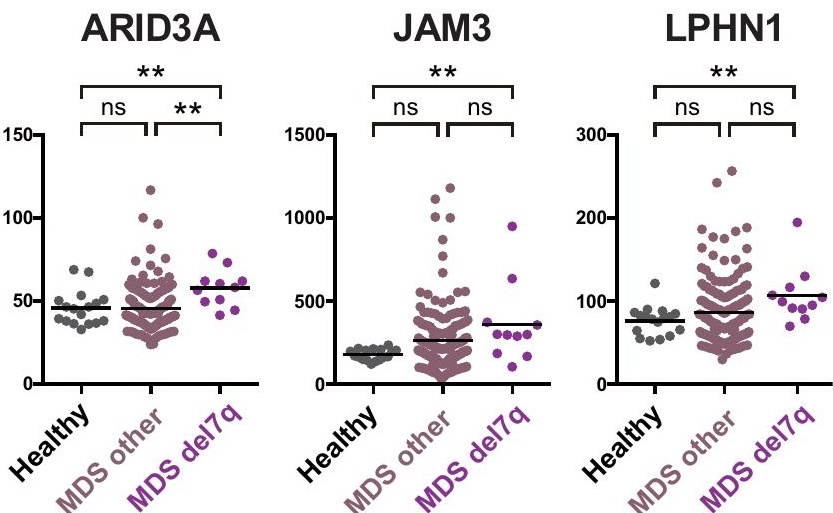




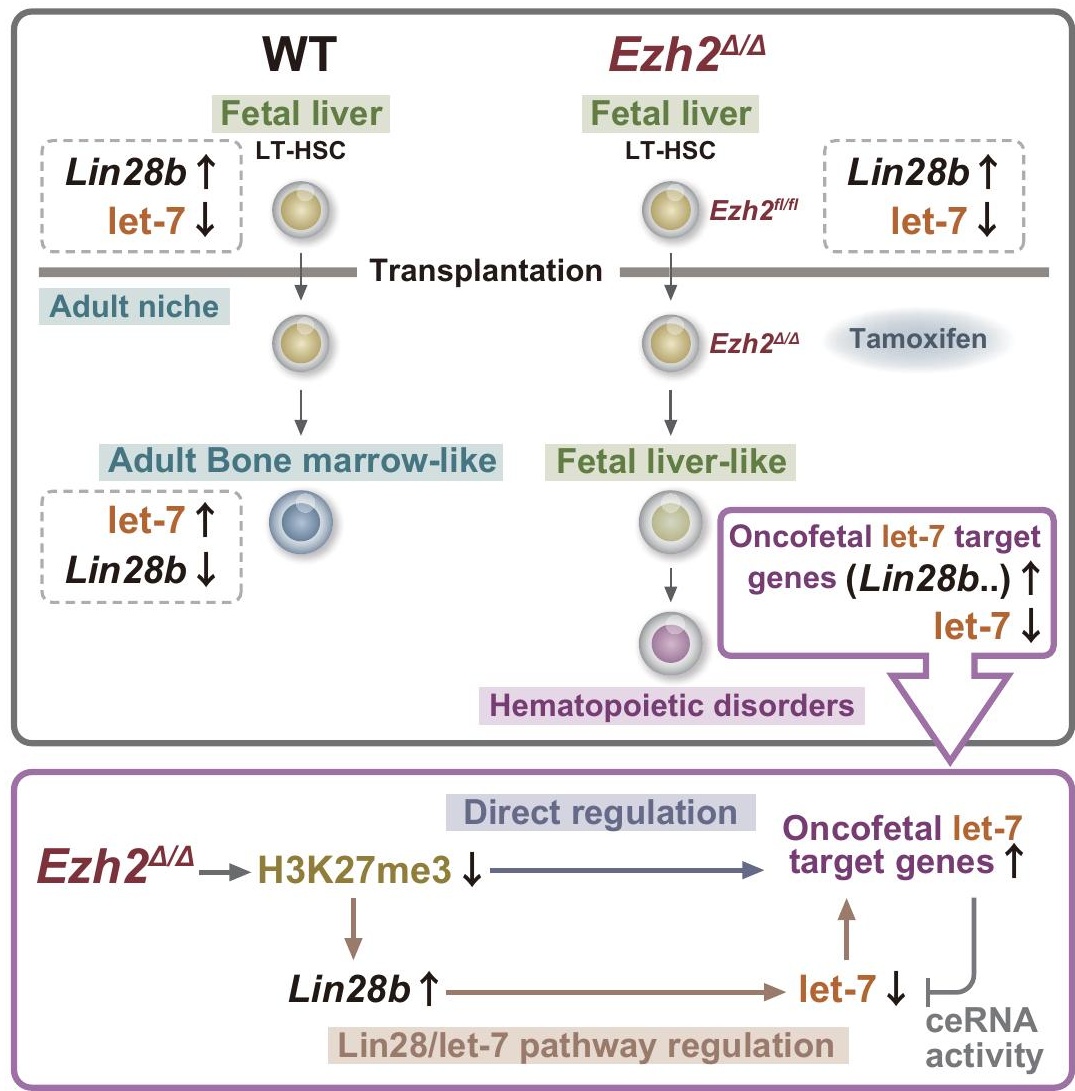


Supplementary Table E1. List of highly expressed genes in both BM Ezh2 $2^{\Delta / \Delta}$ LSK cells and FL LSK cells.

\begin{tabular}{|c|c|c|c|c|c|c|c|c|}
\hline Symbol & $\begin{array}{c}\text { FL / BM } \\
(\log 2)\end{array}$ & $\begin{array}{c}E z h 2^{\Delta / \Delta} \\
\text { / WT } \\
(\log 2)\end{array}$ & Symbol & $\begin{array}{c}\text { FL / BM } \\
(\log 2)\end{array}$ & $\begin{array}{l}E z h 2^{\Delta / \Delta} \\
\text { I WT } \\
(\log 2)\end{array}$ & Symbol & $\begin{array}{c}\text { FL / BM } \\
\text { (log2) }\end{array}$ & $\begin{array}{c}E z h 2^{\Delta / /} \\
/ \text { WT } \\
(\log 2)\end{array}$ \\
\hline 4631405J19Rik & 1.16 & 1.39 & Epb4.115 & 2.68 & 2.54 & Plekhb1 & 1.76 & 1.56 \\
\hline 4933402N22Rik & 1.70 & 1.14 & Evl & 1.81 & 1.14 & Ptk2 & 1.89 & 2.01 \\
\hline 6330403K07Rik & 2.29 & 3.70 & Fbln1 & 3.00 & 1.79 & Rab15 & 2.32 & 1.39 \\
\hline Acbd7 & 2.15 & 2.65 & Fcrl1 & 1.02 & 1.16 & Rag2 & 1.97 & 1.44 \\
\hline Adora3 & 3.92 & 2.41 & Fndc4 & 3.35 & 2.90 & Ramp2 & 3.51 & 1.69 \\
\hline Airn & 2.86 & 1.40 & Fzd9 & 1.17 & 1.71 & Raph1 & 2.97 & 1.49 \\
\hline Arxes2 & 2.08 & 1.06 & Garnl3 & 1.11 & 1.08 & Rpl39l & 1.63 & 5.26 \\
\hline Atp9a & 1.83 & 2.24 & Gcat & 1.27 & 1.28 & Rps4y2 & 1.95 & 1.75 \\
\hline Bank1 & 3.12 & 2.67 & Gm1673 & 1.10 & 1.46 & Rps6ka6 & 3.78 & 2.51 \\
\hline Bdh1 & 1.80 & 2.06 & Gm6816 & 2.01 & 1.77 & Rtkn & 1.75 & 1.13 \\
\hline Beta-s & 2.46 & 1.34 & Gzma & 3.06 & 2.34 & Ryk & 1.05 & 1.23 \\
\hline Bex2 & 1.45 & 2.29 & $\mathrm{Hbb}-\mathrm{b} 1$ & 2.99 & 1.32 & Sept10 & 1.93 & 2.42 \\
\hline Bex4 & 1.62 & 1.24 & $\mathrm{Hbb}-\mathrm{b} 2$ & 2.91 & 1.37 & Sh3gl3 & 3.78 & 1.46 \\
\hline Bfsp2 & 1.69 & 1.33 & Hbb-y & 4.32 & 2.34 & Slc18a1 & 2.03 & 2.80 \\
\hline Brsk1 & 1.04 & 1.03 & Hif3a & 4.44 & 1.10 & Slc29a4 & 1.33 & 1.42 \\
\hline Camkv & 2.10 & 3.18 & Hmga2 & 4.16 & 2.72 & Smoc1 & 1.41 & 2.81 \\
\hline Cbs & 1.49 & 1.65 & $\operatorname{lgf} 2 \mathrm{bp} 3$ & 6.38 & 3.13 & Speer1-ps1 & 1.74 & 1.57 \\
\hline Ccr9 & 2.51 & 3.46 & $\operatorname{lgf} 2 r$ & 1.10 & 1.91 & Speer2 & 1.24 & 1.22 \\
\hline Cdon & 2.40 & 1.64 & $117 r$ & 1.33 & 1.97 & Speer5-ps1 & 1.06 & 1.18 \\
\hline Cgref1 & 1.04 & 1.45 & Jam3 & 1.89 & 1.70 & Speer6-ps1 & 1.42 & 1.48 \\
\hline Chga & 1.07 & 1.41 & Lgr5 & 1.25 & 2.61 & Spire1 & 2.71 & 1.66 \\
\hline Chi3l7 & 4.04 & 1.06 & Lin28b & 8.38 & 2.98 & St3gal6 & 1.70 & 1.34 \\
\hline Ckb & 1.49 & 1.75 & Lphn1 & 1.18 & 1.07 & Tff1 & 4.92 & 1.01 \\
\hline Clip4 & 2.68 & 2.64 & Lrfn3 & 1.69 & 1.14 & Tmeff1 & 2.77 & 2.42 \\
\hline Cnn3 & 2.41 & 2.04 & Lrrc32 & 2.31 & 1.15 & Tmem91 & 2.94 & 1.67 \\
\hline Cpe & 1.42 & 2.64 & Magi1 & 3.76 & 2.35 & Tstd3 & 2.48 & 2.02 \\
\hline Crispld2 & 3.94 & 1.32 & Mfsd2a & 1.10 & 2.52 & Tulp3 & 1.46 & 1.58 \\
\hline D430019H16Rik & 4.72 & 2.47 & MIf1 & 1.60 & 1.42 & Tyro3 & 1.64 & 1.55 \\
\hline Ddx3y & 6.51 & 6.69 & Mrvi1 & 1.48 & 1.72 & Uaca & 1.30 & 1.06 \\
\hline $\mathrm{Ddx} 4$ & 2.20 & 3.43 & Mzb1 & 4.06 & 2.06 & Unc13b & 2.85 & 1.70 \\
\hline Ebi3 & 1.57 & 1.26 & Ninl & 3.18 & 2.32 & Uty & 3.54 & 3.66 \\
\hline Echdc2 & 1.34 & 2.04 & Nsg2 & 1.26 & 1.18 & Wdr65 & 2.98 & 1.20 \\
\hline Eif2s3y & 7.97 & 7.94 & Pdgfa & 1.85 & 1.67 & Zfp781 & 2.79 & 3.38 \\
\hline Elovl2 & 2.89 & 3.63 & Plagl1 & 2.55 & 2.75 & Zmat3 & 1.01 & 1.16 \\
\hline
\end{tabular}

Fold changes of indicated genes in the FL LSK cells relative to BM LSK cells (FL/BM) and those in Ezh2 $2^{\Delta / \Delta}$ BM LSK cells relative to WT BM LSK cells $\left(E z h 2^{\Delta / \Delta} / \mathrm{WT}\right)$ are shown. 
Supplementary Table E2. List of FL-specific let-7 target genes.

\begin{tabular}{|c|c|c|c|c|c|}
\hline Symbol & $\begin{array}{c}\text { FL / BM } \\
(\log 2)\end{array}$ & Symbol & $\begin{array}{c}\text { FL / BM } \\
(\log 2)\end{array}$ & Symbol & $\begin{array}{l}\text { FL / BM } \\
\text { (log2) }\end{array}$ \\
\hline 2310008H09Rik & 1.46 & E2f5 & 1.16 & Pdgfb & 3.02 \\
\hline 2410042D21Rik & 1.56 & Eif2s2 & 1.09 & Pfkfb1 & 2.20 \\
\hline 2700081015Rik & 1.10 & Elovl2 & 2.89 & Pias4 & 1.03 \\
\hline 9930012K11Rik & 1.94 & Eme1 & 1.67 & Pigw & 1.24 \\
\hline Abcb9 & 1.16 & Emid2 & 2.89 & Plxnd1 & 1.89 \\
\hline Acer3 & 1.01 & Enc1 & 1.56 & Pofut1 & 1.01 \\
\hline Ahctf1 & 1.10 & Fam43a & 1.08 & Prr5I & 2.00 \\
\hline Al846148 & 1.15 & Farp1 & 2.92 & Rab15 & 2.32 \\
\hline Alpk3 & 1.18 & Gipc1 & 1.11 & Ralb & 1.08 \\
\hline Ankrd49 & 1.23 & Grb10 & 1.37 & Reep1 & 2.80 \\
\hline Arc & 1.03 & Hecw2 & 4.76 & Runx1t1 & 2.85 \\
\hline Arhgap11a & 1.45 & Hif3a & 4.44 & Sema3f & 1.13 \\
\hline Arhgap28 & 1.16 & Hmga1 & 1.84 & Sema4f & 2.90 \\
\hline Arid3a & 3.56 & Hmga2 & 4.16 & Slc12a9 & 1.02 \\
\hline Arid3b & 1.93 & $\operatorname{lgf} 2 \mathrm{bp} 1$ & 7.97 & Slc24a3 & 1.30 \\
\hline Arl5a & 1.17 & $\operatorname{lgf} 2 \mathrm{bp} 2$ & 5.06 & Smug1 & 1.76 \\
\hline Atad5 & 1.18 & $\operatorname{lgf} 2 b p 3$ & 6.38 & St8sia1 & 2.88 \\
\hline Awat2 & 1.25 & $\lg 2 r$ & 1.10 & Stard13 & 1.24 \\
\hline B3galt4 & 1.08 & Igsf9 & 1.25 & Stx 17 & 1.17 \\
\hline Bcl2l1 & 1.45 & lkzf2 & 1.50 & Syn2 & 2.98 \\
\hline Bcl2/14 & 1.92 & Ipo11 & 1.07 & Tarbp2 & 1.30 \\
\hline $\mathrm{Bmf}$ & 1.36 & Itga4 & 1.07 & Tbkbp1 & 1.06 \\
\hline Btbd3 & 1.98 & Jam3 & 1.89 & Tmc7 & 2.73 \\
\hline Cbx5 & 1.10 & Kctd12b & 1.17 & Tmem110 & 1.34 \\
\hline Ccnd1 & 1.70 & KIhl13 & 2.29 & Tmem177 & 1.23 \\
\hline Ccnyl1 & 1.30 & Lin28b & 8.38 & Trmt11 & 1.01 \\
\hline Ccr7 & 1.13 & Lpar2 & 1.06 & Trove2 & 1.02 \\
\hline Cd200r1 & 1.48 & Lpgat1 & 1.99 & Trp53bp1 & 1.01 \\
\hline Cd2ap & 1.24 & Lphn1 & 1.18 & Tspan18 & 3.25 \\
\hline Cdc14b & 1.65 & Luc7|3 & 1.07 & Ttll4 & 2.20 \\
\hline Cdc34 & 1.62 & Mcts2 & 2.15 & Txn2 & 1.27 \\
\hline Cpd & 3.24 & Mex3a & 1.40 & Ubox5 & 1.05 \\
\hline Crb3 & 2.44 & Mtf2 & 1.38 & Wdr37 & 1.02 \\
\hline Cyyr1 & 3.31 & Mycn & 2.79 & $\mathrm{Xk}$ & 1.53 \\
\hline Dcbld1 & 3.61 & Myh10 & 2.90 & Xkr8 & 1.58 \\
\hline Dgki & 2.72 & Myo7a & 2.36 & Xpot & 1.04 \\
\hline Dicer1 & 1.34 & Ngly1 & 1.35 & Zbtb7b & 1.01 \\
\hline Dna2 & 1.02 & Nid1 & 2.56 & Zcchc3 & 1.96 \\
\hline Dnm1 & 1.47 & Nid2 & 3.20 & Zdhhc12 & 1.39 \\
\hline Dnmt3a & 1.43 & Nme4 & 1.13 & Zfp354a & 2.43 \\
\hline Dnmt3b & 2.72 & Nr6a1 & 2.72 & Zfp462 & 2.53 \\
\hline Dpp3 & 1.16 & Nrtn & 1.42 & Zfp697 & 1.16 \\
\hline Dusp22 & 1.06 & Pabpc4l & 1.43 & Zfp748 & 1.37 \\
\hline Dusp7 & 1.11 & $\mathrm{Pbx} 2$ & 1.25 & Zik1 & 1.21 \\
\hline Dusp9 & 1.48 & Pcdh7 & 1.91 & & \\
\hline
\end{tabular}

Fold changes of indicated genes in the FL LSK cells relative to BM LSK cells (FL/BM) are shown. 
Supplementary Table E3. List of BM-specific PRC2 targets specificaly expressed in FL.

\begin{tabular}{|c|c|c|c|c|c|}
\hline \multirow[b]{2}{*}{ Symbol } & \multicolumn{2}{|c|}{ FL / BM (log2) } & \multirow[b]{2}{*}{ Symbol } & \multicolumn{2}{|c|}{ FL / BM ( $\log 2)$} \\
\hline & H3K27me3 & Expression & & H3K27me3 & Expression \\
\hline 2900092C05Rik & -1.14 & 2.64 & Jam3 & -1.76 & 1.89 \\
\hline Atp9a & -1.02 & 1.83 & Kirrel & -1.06 & 2.55 \\
\hline BC100530 & -1.71 & 9.97 & Ldb2 & -1.20 & 4.04 \\
\hline Bex4 & -1.56 & 1.62 & Lgals2 & -1.06 & 1.43 \\
\hline Cd2ap & -1.10 & 1.24 & $\operatorname{Lin} 28 b$ & -3.59 & 8.38 \\
\hline Cnn3 & -1.66 & 2.41 & Mastl & -1.42 & 1.09 \\
\hline Col18a1 & -1.74 & 5.16 & Mrvi1 & -1.21 & 1.48 \\
\hline Ctnnd1 & -1.14 & 1.00 & Myom1 & -1.01 & 3.11 \\
\hline Cyyr1 & -1.99 & 3.31 & Ngly1 & -1.22 & 1.35 \\
\hline Dcbld1 & -1.09 & 3.61 & Nox4 & -1.55 & 4.11 \\
\hline Ddx3y & -1.42 & 6.51 & Olfr1247 & -1.47 & 2.60 \\
\hline Dyrk4 & -1.31 & 1.65 & P2ry1 & -1.14 & 1.51 \\
\hline Ear1 & -1.26 & 2.16 & Pcdh7 & -1.29 & 1.91 \\
\hline Ecscr & -1.02 & 2.54 & Pde3b & -1.87 & 1.83 \\
\hline Epb4.115 & -1.14 & 2.68 & Pdgfb & -1.55 & 3.02 \\
\hline Evl & -1.50 & 1.81 & Pdgfrl & -1.73 & 2.42 \\
\hline F5 & -1.36 & 2.98 & Plag1 & -1.30 & 2.14 \\
\hline Fam181b & -1.30 & 2.24 & Plxnd1 & -1.32 & 1.89 \\
\hline Fam55d & -1.27 & 1.20 & Rab39 & -1.86 & 3.21 \\
\hline Fbln1 & -1.54 & 3.00 & Ramp2 & -1.79 & 3.51 \\
\hline Fgl1 & -1.04 & 1.49 & Rassf9 & -1.81 & 4.54 \\
\hline Fjx1 & -1.17 & 3.44 & Rtkn & -1.07 & 1.75 \\
\hline Fscn 1 & -1.27 & 3.11 & Sgol2 & -1.06 & 2.10 \\
\hline Fzd9 & -1.46 & 1.17 & Sh3pxd2b & -1.35 & 1.49 \\
\hline Gja1 & -1.25 & 1.46 & Smtnl2 & -1.04 & 1.62 \\
\hline GImn & -1.21 & 1.63 & Sox18 & -1.05 & 4.72 \\
\hline Gpr124 & -1.27 & 3.29 & Spats2 & -1.36 & 5.16 \\
\hline Gpx3 & -1.69 & 2.62 & Stard13 & -1.01 & 1.24 \\
\hline Grap & -1.12 & 4.11 & Sufu & -1.06 & 1.41 \\
\hline Grrp1 & -1.08 & 5.16 & Tek & -1.13 & 1.76 \\
\hline Gzma & -1.08 & 3.06 & Tekt1 & -1.22 & 3.76 \\
\hline $\mathrm{Hbb}-\mathrm{b} 1$ & -1.75 & 2.99 & Tmem91 & -1.18 & 2.94 \\
\hline $\mathrm{Hbb}-\mathrm{b} 2$ & -1.75 & 2.91 & Tnfrsf25 & -1.34 & 6.80 \\
\hline Hif3a & -1.43 & 4.44 & Trmt11 & -1.04 & 1.01 \\
\hline Hist1h2bb & -1.54 & 1.39 & Tspan8 & -1.42 & 3.12 \\
\hline Hmga2 & -2.06 & 4.16 & Ttpa & -1.70 & 2.81 \\
\hline $\operatorname{lgf1}$ & -1.45 & 4.54 & Ubtd1 & -1.58 & 1.01 \\
\hline $\operatorname{lgf} 2 b p 1$ & -2.06 & 7.97 & Ubtd2 & -1.63 & 1.57 \\
\hline $\operatorname{lgf} 2 b p 2$ & -1.94 & 5.06 & Zfp354a & -1.60 & 2.43 \\
\hline $\operatorname{lgf} 2 b p 3$ & -3.29 & 6.38 & Zfp711 & -1.57 & 5.64 \\
\hline II5ra & -1.31 & 1.73 & & & \\
\hline
\end{tabular}

$\square$ let-7 target genes

Fold enrichment values of H3K27me3 against the input signals (ChIP/Input) (TSS $\pm 2.0 \mathrm{~kb}$ of the genes) between FL and BM LSK cells are shown in the left column. Fold changes of indicated genes in the FL LSK cells relative to BM LSK cells are shown in the right column. 


\section{EIMMo putative let-7 target genes}

\section{Putative let-7 target gene set was generated using a software avai}

Gene Symbol Gene Definition

0610007P08Rik RAD26L hypothetical protein isoform 2

1110005A03Rik hypothetical protein LOC74319

1110018G07Rik hypothetical protein LOC68497

1110037F02Rik hypothetical protein LOC66185

1110054005Rik hypothetical protein LOC66209

1110059G10Rik hypothetical protein LOC66202

1200016B10Rik hypothetical protein LOC66875

1500003003Rik calcium binding protein P22

1600012H06Rik hypothetical protein LOC67912

1600014C10Rik hypothetical protein LOC72244

1700017B05Rik septin 14

1700029H14Rik hypothetical protein LOC66501 isoform 2

1700037H04Rik hypothetical protein LOC67326

1700052N19Rik hypothetical protein LOC73419

1700066B19Rik hypothetical protein LOC73449

1810013L24Rik hypothetical protein LOC69053

1810031K17Rik hypothetical protein LOC69171

2010002N04Rik putative small membrane protein NID67

2010317E24Rik hypothetical protein LOC72080

2200002K05Rik hypothetical protein LOC69137

2310008H09Rik hypothetical protein LOC66356

2310014L17Rik hypothetical protein LOC381845

2310022A10Rik hypothetical protein LOC66367 isoform 2

2410002I01Rik hypothetical protein LOC78777

2410042D21Rik hypothetical protein LOC72425

2700081015Rik hypothetical protein LOC108899

2810030E01Rik hypothetical protein LOC72668

2810407C02Rik selenoprotein T

2810474019Rik hypothetical protein LOC67246

3110056003Rik signal peptide peptidase-like 2B

3110082I17Rik hypothetical protein LOC73212

3200002M19Rik hypothetical protein LOC75430

4832428D23Rik hypothetical protein LOC403183

4930444G20Rik hypothetical protein LOC114671 
4930555I21Rik hypothetical protein LOC78806

4931408C20Rik hypothetical protein LOC210940

4931428F04Rik hypothetical protein LOC74356

4933403F05Rik hypothetical protein LOC108654

5031439G07Rik hypothetical protein LOC223739

5430435G22Rik hypothetical protein LOC226421

5930434B04Rik hypothetical protein LOC381356

6230409E13Rik hypothetical protein LOC76132

6330416G13Rik hypothetical protein LOC230279

6330578E17Rik hypothetical protein LOC76178

6430571L13Rik hypothetical protein LOC235599

6720456H20Rik hypothetical protein LOC218989

8430427H17Rik hypothetical protein LOC329540

9130023H24Rik hypothetical protein LOC319792

9930012K11Rik hypothetical protein LOC268759 isoform 2

A130022J15Rik RIKEN cDNA A130022J15

A1cf APOBEC1 complementation factor

A530054K11Rik hypothetical protein LOC212281

A830007P12Rik hypothetical protein LOC227612

Abcb9 ATP-binding cassette sub-family B member 9

Abcc10 ATP-binding cassette sub-family C member 10

Abcc5 ATP-binding cassette sub-family $C$ member 5

Abcg1 ATP-binding cassette subfamily $G$ member 1

Abcg4 ATP-binding cassette sub-family G (WHITE)

Abi3 $\mathrm{ABI}$ gene family member 3

Abl2 v-abl Abelson murine leukemia viral oncogene 2

Acer1 $\mathrm{N}$-acylsphingosine amidohydrolase 3

Acer2 alkaline ceramidase 2

Acer3 phytoceramidase alkaline

Acot11 acyl-CoA thioesterase 11

Acpp acid phosphatase prostate isoform 1

Acss1 acetyl-CoA synthetase 2-like

Acta1 actin alpha 1 skeletal muscle

Actr2 actin-related protein 2

Actr5 ARP5 actin-related protein 5 homolog

Acvr1b activin A receptor type $1 \mathrm{~B}$

Acvr2a activin receptor IIA precursor

Adam8 a disintegrin and metallopeptidase domain 8

Adamts12 a disintegrin-like and metalloprotease 
Adamts14 a disintegrin-like and metallopeptidase

Adamts15 a disintegrin-like and metalloprotease

Adamts5 a disintegrin-like and metalloprotease

Adamts8 a disintegrin-like and metalloprotease

AdamtsI4 ADAMTS-like 4

Adipor2 adiponectin receptor 2

Adra1b adrenergic receptor alpha $1 b$

Adra2b adrenergic receptor alpha $2 b$

Adrb1 adrenergic receptor beta 1

Adrb2 adrenergic receptor beta 2

Adrb3 adrenergic receptor beta 3

Aen interferon stimulated exonuclease gene 20-like

AF366264 SUMO-1 specific protease 4

Afap1l2 actin filament associated protein 1-like 2

Aff4 AF4/FMR2 family member 4

Agpat6 lysophosphatidic acid acyltransferase zeta

Agphd1 hypothetical protein LOC235386

Agtr1a angiotensin II receptor type 1a

Ahctf1 AT hook containing transcription factor 1

Ahr aryl-hydrocarbon receptor

Al464131 hypothetical protein LOC329828

Al593442 hypothetical protein LOC330941 isoform 2

Al846148 hypothetical protein LOC68229

Aida axin interaction partner and dorsalization

Akap6 A kinase (PRKA) anchor protein 6

Akap8 A kinase anchor protein 8

Akt1 thymoma viral proto-oncogene 1

Aldh6a1 aldehyde dehydrogenase family 6 subfamily A1

Alkbh4 alkB alkylation repair homolog 4

Alox5 arachidonate 5-lipoxygenase

Alpk3 myocyte induction differentiation originator

Amot angiomotin

Angptl2 angiopoietin-like 2

Ankfy1 ankyrin repeat and FYVE domain containing 1

Ankib1 ankyrin repeat and IBR domain containing 1

Ankrd10 ankyrin repeat domain 10

Ankrd49 ankyrin repeat domain 49

Ankrd52 ankyrin repeat domain 52

Ankrd6 ankyrin repeat domain 6 
Anxa8 annexin A8

Aoc3 amine oxidase copper containing 3

Ap1s1 adaptor protein complex AP-1 sigma 1

Ap3d1 adaptor-related protein complex 3 delta 1

Ap3m1 adaptor-related protein complex 3 mu 1 subunit

Apba1 amyloid beta (A4) precursor protein binding

Apbb3 amyloid beta (A4) precursor protein-binding

Apc2 adenomatosis polyposis coli 2

Aplp2 amyloid beta (A4) precursor-like protein 2

Aqp4 aquaporin 4

Arc activity regulated cytoskeletal-associated

Arfgef2 ADP-ribosylation factor guanine

Arhgap11a Rho GTPase activating protein 11A

Arhgap19 Rho GTPase activating protein 19

Arhgap20 Rho GTPase activating protein 20

Arhgap28 Rho GTPase activating protein 28

Arhgap29 Rho GTPase activating protein 29

Arhgef12 Rho guanine nucleotide exchange factor 12

Arhgef15 Rho guanine nucleotide exchange factor (GEF) 15

Arhgef6 Rac/Cdc42 guanine nucleotide exchange factor 6

Arhgef9 Cdc42 guanine nucleotide exchange factor (GEF)

Arid1b AT rich interactive domain 1B (Swi1 like)

Arid3a dead ringer homolog 1

Arid3b dead ringer homolog 2

Arid3c AT rich interactive domain 3C

Arih1 ariadne ubiquitin-conjugating enzyme E2 binding

Arl4d ADP-ribosylation factor-like 4D

Arl5a ADP-ribosylation factor-like 5

Armc2 armadillo repeat containing 2

Armc8 armadillo repeat containing 8

Arpp19 cAMP-regulated phosphoprotein 19

Arrdc4 arrestin domain containing 4 isoform 2

Arsg Arylsulfatase G

Arv1 ARV1 homolog

Asah2 N-acylsphingosine amidohydrolase 2

Asap1 development and differentiation enhancing

Asb8 ankyrin repeat and SOCS box-containing protein

Ascl1 achaete-scute complex homolog 1

Ash1l absent small or homeotic discs 1 
Asnsd1 asparagine synthetase domain containing 1

Asph aspartyl beta-hydroxylase isoform 1

Atad3a AAA-ATPase TOB3

Atad5 ATPase family AAA domain containing 5

Atf7ip activating transcription factor 7 interacting

Atg4b autophagin 1

Atp10b ATPase class $V$ type 10B

Atp2a2 ATPase $\mathrm{Ca}++$ transporting cardiac muscle slow

Atp6v1b2 vacuolar H+ATPase B2

Atp8b4 ATPase class I type 8B member 4

Atpaf1 ATP synthase mitochondrial F1 complex assembly

Atxn1l ataxin 1-like

Atxn3 ataxin 3

Atxn7 ataxin 7

Aven apoptosis caspase activation inhibitor

AW549877 hypothetical protein LOC106064

Awat2 diacylglycerol O-acyltransferase 2-like 4

B3galt1 UDP-Gal:betaGIcNAc beta

B3galt4 UDP-Gal:betaGalNAc beta

B3gat1 beta-13-glucuronyltransferase 1

B3gat3 beta-13-glucuronyltransferase 3

B3gnt1 beta-13-N-acetylglucosaminyltransferase bGnT-6

B3gnt7 UDP-GlcNAc:betaGal

B630005N14Rik hypothetical protein LOC101148

B930041F14Rik hypothetical protein LOC230991

Bach1 BTB and CNC homology 1

Bahcc1 BAH domain and coiled-coil containing 1

Bahd1 bromo adjacent homology domain containing 1

Baiap2 brain-specific angiogenesis inhibitor

Batf basic leucine zipper transcription factor-like

BC006779 hypothetical protein LOC229003

BC068157 hypothetical protein LOC73072

Bcap29 B-cell receptor-associated protein 29

Bcl2l1 Bcl2-like 1

Bcl2I13 BCL2-like 13

$\mathrm{Bcl}$ 2I14 apoptosis regulator BCL-G

Bcl7a B-cell CLL/lymphoma 7A

Best2 bestrophin 2

Bicd1 bicaudal D homolog 1 isoform 1 
Bik Bcl2-interacting killer

Bin3 bridging integrator 3

Bmf Bcl2 modifying factor

Braf Braf transforming

Brcc3 BRCA1/BRCA2-containing complex subunit 3

Brpf3 bromodomain and PHD finger containing 3

Brsk2 brain-selective kinase 2 isoform alpha

Brwd1 bromodomain and WD repeat domain containing 1

Bsn bassoon protein

Bsnd Bartter syndrome infantile with sensorineural

Btbd3 BTB/POZ domain containing protein 3 isoform 2

Btf3|4 basic transcription factor 3-like 4

Btg2 B-cell translocation gene 2 anti-proliferative

Bzw1 basic leucine zipper and W2 domains 1

Bzw2 basic leucine zipper and W2 domains 2

C2 complement component 2

C230081A13Rik NKF3 kinase family member

C2cd2 hypothetical protein LOC207781

C2cd2I transmembrane protein 24

C330019G07Rik hypothetical protein LOC215476

Cacna1e calcium channel voltage-dependent $\mathrm{R}$ type

Cacnb2 calcium channel voltage-dependent beta 2

Cacnb4 calcium channel voltage-dependent beta 4

Cadm3 immunoglobulin superfamily member 4B

Cald1 caldesmon 1

Calm1 calmodulin 1

Calm4 calmodulin 4

Camk4 calcium/calmodulin-dependent protein kinase IV

Cap1 CAP adenylate cyclase-associated protein 1

Cap2 CAP adenylate cyclase-associated protein 2

Capn1 calpain 1 large subunit

Car2 carbonic anhydrase 2

Car7 carbonic anhydrase 7

Casc 3 cancer susceptibility candidate 3

Cask calcium/calmodulin-dependent serine protein

Caskin1 CASK interacting protein 1

Casp3 caspase 3

Casp7 caspase 7

Cat catalase 
Cbln4 cerebellin 4 precursor

Cbx2 chromobox homolog 2

Cbx5 chromobox homolog 5

Cc2d1a coiled-coil and C2 domain containing 1A

Ccdc25 coiled-coil domain containing 25

Ccdc72 coiled-coil domain containing 72

Ccdc93 coiled-coil domain containing 93 isoform a

Ccnd1 cyclin D1

Ccnd2 cyclin D2

Ccnh cyclin $\mathrm{H}$

Ccnj cyclin J

Ccny cyclin fold protein 1

Ccnyl1 cyclin Y-like 1

Ccr7 chemokine (C-C motif) receptor 7

Cd164 CD164 antigen

Cd200 Cd200 antigen

Cd200r1 CD200 receptor 1

Cd209e Cd209e antigen

Cd276 CD276 antigen

Cd2ap CD2-associated protein

Cd34 CD34 antigen isoform 1

Cd83 CD83 antigen

Cd86 CD86 antigen

Cd93 CD93 antigen

Cdan1 congenital dyserythropoietic anemia type I

Cdc14b CDC14 cell division cycle 14 homolog $B$ isoform

Cdc25a cell division cycle 25 homolog A

Cdc34 cell division cycle 34 homolog

Cdca5 cell division cycle associated 5

Cdh22 cadherin 22

Colh26 cadherin-like 26

Cdk5r2 cyclin-dependent kinase 5 regulatory subunit 2

Cdk6 cyclin-dependent kinase 6

Cdv3 carnitine deficiency-associated gene expressed

Cdyl chromodomain protein $Y$ chromosome-like

Cebpd CCAAT/enhancer binding protein delta

Cecr 5 cat eye syndrome chromosome region candidate 5

Cecr6 cat eye syndrome chromosome region candidate 6

Cend1 cell cycle exit and neuronal differentiation 1 
Cep120 coiled-coil domain containing 100

Cep135 centrosomal protein 135

Cep164 centrosomal protein 164

Cep57 translokin

Cer1 cerberus 1

Cercam cerebral endothelial cell adhesion molecule 1

Cggbp1 CGG triplet repeat binding protein 1

Cgnl1 cingulin-like 1

Chchd3 coiled-coil-helix-coiled-coil-helix domain

Chd4 chromodomain helicase DNA binding protein 4

Chd9 chromodomain helicase DNA binding protein 9

Chka choline kinase alpha isoform 1

Chn2 beta chimerin

Chrd chordin

Chrm1 cholinergic receptor muscarinic 1

Chrna6 cholinergic receptor nicotinic alpha

Chrnb2 neuronal nicotinic acetylcholine receptor beta

Chst10 carbohydrate sulfotransferase 10

Chst3 carbohydrate (chondroitin 6/keratan)

Chsy3 chondroitin sulfate synthase 3

Clec16a C-type lectin domain family 16 member A

Clec4a1 C-type lectin domain family 4 member a1

CIp1 ATP/GTP-binding protein

Clpb suppressor of $\mathrm{K}+$ transport defect 3

Clrn3 clarin 3

Cmah cytidine monophospho- $\mathrm{N}$-acetylneuraminic acid

Cnih cornichon homolog

Cnn1 calponin 1

Cnnm4 cyclin M4

Cnp 2'3'-cyclic nucleotide 3' phosphodiesterase

Cntn2 contactin 2

Coil coilin

Col10a1 procollagen type $X$ alpha 1

Col14a1 procollagen type XIV alpha 1

Col15a1 procollagen type $\mathrm{XV}$

Col19a1 procollagen type XIX alpha 1

Col1a2 procollagen type I alpha 2

Col23a1 collagen type XXIII alpha 1

Col24a1 procollagen type XXIV alpha 1 
Col27a1 procollagen type XXVII alpha 1

Col4a1 collagen type IV alpha 1

Col4a2 procollagen type IV alpha 2

Col5a2 procollagen type $\mathrm{V}$ alpha 2

Col9a1 collagen type IX alpha 1

Col9a3 procollagen type IX alpha 3

Colec10 collectin sub-family member 10

Cops7b COP9 (constitutive photomorphogenic) homolog

Corola coronin actin binding protein $1 \mathrm{~A}$

Cpa4 carboxypeptidase A4

Cpd carboxypeptidase D

Cpeb3 cytoplasmic polyadenylation element binding

Cpsf4 cleavage and polyadenylation specific factor 4

Crb3 crumbs homolog 3

Crct1 cysteine-rich C-terminal 1

Creg1 cellular repressor of E1A-stimulated genes 1

Creg2 cellular repressor of E1A-stimulated genes 2

Crk v-crk sarcoma virus CT10 oncogene homolog

Crp C-reactive protein pentraxin-related

Crtac1 cartilage acidic protein 1

Crtam cytotoxic and regulatory $\mathrm{T}$ cell molecule

Crtap cartilage associated protein

Cry2 cryptochrome 2 (photolyase-like)

Crybg3 hypothetical protein LOC224273

Csnk1e casein kinase 1 epsilon

Csnk1g1 casein kinase 1 gamma 1

Csnk2a1 casein kinase 2 alpha 1 polypeptide

Csnk2a2 casein kinase 2 alpha prime polypeptide

Csrnp2 cDNA sequence BC035295

Ctage5 CTAGE family member 5

Ctbs chitobiase di-N-acetyl-

Ctdspl2 CTD (carboxy-terminal domain RNA polymerase II

Ctns cystinosis nephropathic

Ctsb cathepsin B preproprotein

Cul2 cullin 2

Cxcr2 interleukin 8 receptor beta

Cyb561d1 cytochrome b-561 domain containing 1 isoform 2

Cyld ubiquitin carboxyl-terminal hydrolase CYLD

Cyp2c70 cytochrome P450 family 2 subfamily c 
Cyp46a1 cytochrome P450 family 46 subfamily a

Cyp4f13 cytochrome $P 450$ family 4 subfamily $f$

Cyth1 pleckstrin homology $\operatorname{Sec} 7$ and coiled-coil

Cyyr 1 cysteine and tyrosine-rich protein 1

D11Wsu99e hypothetical protein LOC28081

D14Abb1e retinoblastoma-associated protein 140 isoform 2

D2hgdh D-2-hydroxyglutarate dehydrogenase

Dab1 disabled homolog 1 isoform 1

Dagla diacylglycerol lipase alpha

Dapk1 death associated protein kinase 1

Dbc1 deleted in bladder cancer chromosome region

Dcaf10 WD repeat domain 32

Dcaf15 hypothetical protein LOC212123

Dcaf8 H326

Dcbld1 discoidin CUB and LCCL domain containing 1

Dclre1b DNA cross-link repair 1B PSO2 homolog isoform

Dcp1a MAD homolog 4 interacting transcription

Dcun1d3 DCN1 defective in cullin neddylation 1 domain

Dcun1d4 DCN1 defective in cullin neddylation 1 domain

Ddi2 DNA-damage inducible protein 2

Ddit4I DNA-damage-inducible transcript 4-like

Ddn dendrin

Ddo D-aspartate oxidase

Ddost dolichyl-di-phosphooligosaccharide-protein

Ddx19a Ddx19-like protein

Ddx19b DDX19 homolog

Ddx26b DEAD/H (Asp-Glu-Ala-Asp/His) box polypeptide

Decr1 24-dienoyl CoA reductase 1 mitochondrial

Dennd1a DENN/MADD domain containing 1A

Dennd3 DENN/MADD domain containing 3

Dffa DNA fragmentation factor alpha subunit isoform

Dgcr14 DiGeorge syndrome critical region protein 14

Dgki diacylglycerol kinase iota

Dhtkd1 dehydrogenase E1 and transketolase domain

Dhx8 DEAH (Asp-Glu-Ala-His) box polypeptide 8

Dicer1 dicer1

Dkk3 dickkopf homolog 3

DId dihydrolipoamide dehydrogenase

Dlgap1 discs large homolog-associated protein 1 
Dlst dihydrolipoamide S-succinyltransferase (E2

Dmp1 dentin matrix protein 1

Dmrta1 doublesex and mab-3 related transcription factor

Dmrtc2 doublesex and mab-3 related transcription factor

Dna2 DNA replication helicase 2 homolog

Dnaja2 DnaJ (Hsp40) homolog subfamily A member 2

Dnaja4 heat shock protein DNAJ-like 4

Dnajb9 DnaJ (Hsp40) homolog subfamily B member 9

Dnajc1 DnaJ (Hsp40) homolog subfamily C member 1

Dnajc18 DnaJ (Hsp40) homolog subfamily C member 18

Dnajc27 rab and DnaJ domain containing

Dnalc1 axonemal dynein light chain 1

Dnase1l2 deoxyribonuclease 1-like 2

Dnlz hypothetical protein LOC52838

Dnm1 dynamin

Dnm3 dynamin 3

Dnmt3a DNA methyltransferase 3A isoform 2

Dnmt3b DNA methyltransferase 3B isoform 2

Dock3 dedicator of cyto-kinesis 3

Dot1l DOT1-like histone H3 methyltransferase

Dpagt1 UDP-N-acetylglucosamine-dolichyl-phosphate

Dpf2 D4 zinc and double PHD fingers family 2

Dpp3 dipeptidyl peptidase III

Dsc3 desmocollin 3

Dst dystonin isoform a

Dstyk receptor interacting protein kinase 5

Dtx4 deltex 4 homolog

Dusp1 dual specificity phosphatase 1

Dusp22 dual specificity phosphatase TS-DSP2 isoform b

Dusp4 dual specificity phosphatase 4

Dusp6 dual specificity phosphatase 6

Dusp7 dual specificity phosphatase 7

Dusp9 dual specificity phosphatase 9

Dyrk1a dual-specificity tyrosine-(Y)-phosphorylation

Dzip1 DAZ interacting protein 1

Dzip1I DAZ interacting protein 1-like

E130306D19Rik hypothetical protein LOC230098

E2f2 E2F transcription factor 2

E2f3 E2F transcription factor 3 
E2f5 E2F transcription factor 5

E2f6 E2F transcription factor 6

E2f7 E2F transcription factor 7

Ebag9 estrogen receptor-binding fragment-associated

Ebf4 early B-cell factor 4

Ecm2 extracellular matrix protein 2

Edc3 enhancer of mRNA decapping 3

Edem1 ER degradation enhancer mannosidase alpha-like

Edem3 ER degradation enhancer mannosidase alpha-like

Edn1 endothelin 1

Eea1 early endosome antigen 1

Efhd2 EF hand domain containing 2

Efnb3 ephrin B3

Efr3a RIKEN cDNA C920006C10

EgIn2 EGL nine homolog 2

Ehhadh L-specific multifunctional beta-oxdiation

Eif1ad eukaryotic translation initiation factor $1 \mathrm{~A}$

Eif2c4 Piwi/Argonaute family protein melF2C4

Eif2s2 eukaryotic translation initiation factor 2

Eif4e1b eukaryotic translation initiation factor 4E

Eif4g2 eukaryotic translation initiation factor 4

Eif5a2 eukaryotic translation initiation factor 5A2

Eif5b eukaryotic translation initiation factor $5 B$

Elac1 elaC homolog 1

Elavl1 ELAV (embryonic lethal abnormal vision

Elk1 ELK1 member of ETS oncogene family

Elovl2 elongation of very long chain fatty acids-like

Elovl4 elongation of very long chain fatty acids-like

Elp2 signal transducer and activator of transcription

Eme1 essential meiotic endonuclease 1 homolog 1

Emid2 EMI domain containing 2

Enc1 ectodermal-neural cortex 1

Entpd7 ectonucleoside triphosphate diphosphohydrolase

Epb4.1 erythrocyte protein band 4.1

Epha3 Eph receptor A3

Epha4 Eph receptor A4

Epha7 Eph receptor A7 isoform 1

Eppk1 epiplakin 1

Eps8I3 epidermal growth factor receptor pathway 
Erc2 ELKS/RAB6-interacting/CAST family member 2

Ercc2 excision repair cross-complementing rodent

Ercc6 excision repair cross-complementing rodent

Ereg epiregulin

Ergic1 endoplasmic reticulum-golgi intermediate

Ergic2 PTX1 protein isoform 1

Ero1l ERO1-like

Ero1lb endoplasmic oxidoreductase 1 beta

Esam endothelial cell-specific adhesion molecule

Espl1 extra spindle poles-like 1

Esyt2 chr2 synaptotagmin

Etnk2 ethanolamine kinase 2

Exoc5 exocyst complex component 5

Ext2 exostosin 2

Ezh2 enhancer of zeste homolog 2

Fam108c hypothetical protein LOC70178

Fam113b hypothetical protein LOC239647

Fam115a hypothetical protein LOC77574

Fam118a hypothetical protein LOC73225 isoform b

Fam123c hypothetical protein LOC211383

Fam125b hypothetical protein LOC72543

Fam126b hypothetical protein LOC213056

Fam135a hypothetical protein LOC68187

Fam154b hypothetical protein LOC330577

Fam174a transmembrane protein 157

Fam175b hypothetical protein LOC109359

Fam178a hypothetical protein LOC226151

Fam184b hypothetical protein LOC58227

Fam189a1 hypothetical protein LOC70638

Fam198b hypothetical protein LOC68659

Fam40b hypothetical protein LOC320609 isoform b

Fam43a hypothetical protein LOC224093

Fam55b hypothetical protein LOC78252

Fam83g hypothetical protein LOC69640

Fam96a family with sequence similarity 96 member $A$

Fancc Fanconi anemia complementation group $C$

Farp1 FERMRhoGEF (Arhgef) and pleckstrin domain

Fas Fas (TNF receptor superfamily member)

FasI Fas ligand (TNF superfamily member 6) 
Fat4 FAT tumor suppressor homolog 4

Fbln5 fibulin 5

Fbxl14 F-box and leucine-rich repeat protein 14

Fbxo30 F-box protein 30

Fbxo32 F-box protein 32

Fbxo41 F-box protein 41

Fbxo42 F-box protein 42

Fbxw11 F-box and WD-40 domain protein 11

Fem1a feminization 1 homolog a

Fga fibrinogen alpha polypeptide isoform 1

Fgd6 FYVE RhoGEF and PH domain containing 6

Fgf14 fibroblast growth factor 14 isoform b

Fgf16 fibroblast growth factor 16

Fgf4 fibroblast growth factor 4

Fgf5 fibroblast growth factor 5

Fgfbp1 fibroblast growth factor binding protein 1

Fign fidgetin

Fkrp fukutin related protein

Fktn fukutin

Flad1 FAD-synthetase

Fmn1 formin 1 isoform 1

Fn3k fructosamine 3 kinase isoform $b$

Fndc3a fibronectin type III domain containing $3 a$

Fndc3b FAD104

Fnip1 folliculin interacting protein 1

Foxk1 forkhead box K1

Foxk2 forkhead box K2

Fpr1 formyl peptide receptor 1

Fras1 Fras1 protein

Frem2 Fras1 related extracellular matrix protein 2

Frk fyn-related kinase

Frmd4b GRP1-binding protein GRSP1

Frmd5 FERM domain containing 5

Frs2 fibroblast growth factor receptor substrate 2

Fuk fucokinase isoform 2

Fut9 fucosyltransferase 9

Gabpa GA repeat binding protein alpha

Gabpb2 GA repeat binding protein beta 2

Gabra6 gamma-aminobutyric acid (GABA-A) receptor 
Gabrb2 gamma-aminobutyric acid (GABA-A) receptor

Gabrd gamma-aminobutyric acid (GABA-A) receptor

Gad1 glutamic acid decarboxylase 1

Gad2 glutamic acid decarboxylase 2

Galc galactosylceramidase

Gale galactose-4-epimerase UDP

Galm galactose mutarotase

Galnt1 UDP-N-acetyl-alpha-D-galactosamine:polypeptide

Galnt12 UDP-N-acetyl-alpha-D-galactosamine:polypeptide

Galnt2 UDP-N-acetyl-alpha-D-galactosamine:polypeptide

Gata1 GATA binding protein 1

Gatm L-arginine:glycine amidinotransferase

Gcap14 granule cell antiserum positive 14 isoform 2

Gcnt1 glucosaminyl ( $\mathrm{N}$-acetyl) transferase 1 core 2

Gcnt3 glucosaminyl (N-acetyl) transferase 3 mucin

Gdap1l1 ganglioside-induced differentiation-associated

Gdap2 ganglioside-induced

Gdf6 growth differentiation factor 6

Gdpd1 glycerophosphodiester phosphodiesterase domain

Gfod1 glucose-fructose oxidoreductase domain

Gfpt1 glutamine fructose-6-phosphate transaminase 1

Gga3 golgi associated gamma adaptin ear containing

Ggps1 geranylgeranyl diphosphate synthase 1

Ggta1 glycoprotein galactosyltransferase alpha 13

Ghr growth hormone receptor isoform 2 precursor

Gigyf1 PERQ amino acid rich with GYF domain 1

Gipc1 GIPC PDZ domain containing family member 1

Gk5 glycerol kinase 5 (putative)

GIce D-glucuronyl C5-epimerase

GIrx glutaredoxin

Gltpd1 glycolipid transfer protein domain containing 1

Gm1568 hypothetical protein LOC380768

Gm16515 transcript expressed during hematopoiesis 2

Gm5134 solute carrier family 5 (sodium/glucose

Gm5567 hypothetical protein LOC434008

Gm5878 hypothetical protein LOC545861

Gnaq guanine nucleotide binding protein alpha q

Gng5 guanine nucleotide binding protein (G protein)

Gnpda1 glucosamine-6-phosphate deaminase 1 
Gnptab N-acetylglucosamine-1-phosphate transferase

Gns glucosamine (N-acetyl)-6-sulfatase

Golga4 golgi autoantigen golgin subfamily a 4

Golph3 golgi phosphoprotein 3

Golt1b golgi transport 1 homolog B

Gpaa1 GPI anchor attachment protein 1

Gpatch2 $\mathrm{G}$ patch domain containing 2

Gpatch3 $\mathrm{G}$ patch domain containing 3

Gpr123 G protein-coupled receptor 123

Gpr132 G protein-coupled receptor 132

Gpr137 G protein-coupled receptor 137

Gpr137b transmembrane 7 superfamily member 1

Gpr156 G protein-coupled receptor 156

Gpr157 G protein-coupled receptor 157

Gpr165 G protein-coupled receptor 165

Gpr20 G protein-coupled receptor 20

Gpr45 G protein-coupled receptor 45

Gpr63 G protein-coupled receptor 63

Gprc5a G protein-coupled receptor family C group 5

Gpx7 glutathione peroxidase 7

Gramd1c GRAM domain containing 1C

Gramd3 GRAM domain containing 3

Grb10 growth factor receptor bound protein 10

Greb1 Greb1 protein

Greb1l cDNA sequence AK220484

Grhl2 transcription factor CP2-like 3

Gria3 glutamate receptor ionotropic AMPA3 precursor

Grid2ip glutamate receptor ionotropic delta 2 (Grid2)

Grik2 glutamate receptor ionotropic kainate 2

Grk1 G protein-coupled receptor kinase 1

Grpel1 GrpE-like 1 mitochondrial

Grpel2 GrpE-like 2 mitochondrial

Gsdmc gasdermin C1

Gsdmc3 gasdermin C3

Gsg1l GSG1-like

Gspt1 G1 to S phase transition 1

Gsr glutathione reductase 1 precursor

Gtf3c2 general transcription factor IIIC polypeptide

Gucy1a3 guanylate cyclase 1 soluble alpha 3 
Gucy2f guanylate cyclase $2 f$

Gusb glucuronidase beta

Gxylt1 glycosyltransferase 8 domain containing 3

Gyk glycerol kinase isoform 2

Gypc glycophorin C

H6pd hexose-6-phosphate dehydrogenase (glucose

Habp4 hyaluronic acid binding protein 4

Hand1 heart and neural crest derivatives expressed

Hap1 huntingtin-associated protein 1

Hapln2 hyaluronan and proteoglycan link protein 2

Has2 hyaluronan synthase 2

Hdac10 histone deacetylase 10

Hdgfrp3 hepatoma-derived growth factor related protein

Hdlbp high density lipoprotein binding protein

Hdx highly divergent homeobox

Heatr5a HEAT repeat containing 5A

Hectd2 HECT domain containing 2

Hecw1 HECT C2 and WW domain containing E3 ubiquitin

Hecw2 HECT C2 and WW domain containing E3 ubiquitin

Hes2 hairy and enhancer of split 2

Hey2 hairy/enhancer-of-split related with YRPW motif

Hic2 hypermethylated in cancer 2

Hif1an hypoxia-inducible factor 1 alpha subunit

Hif3a hypoxia inducible factor 3 alpha subunit

Hist3h2ba histone cluster $3 \mathrm{H} 2 \mathrm{ba}$

Hk2 hexokinase 2

HIf hepatic leukemia factor

Hmg20a high mobility group 20A

Hmga1 high mobility group AT-hook 1

Hmga2 high mobility group AT-hook 2

Hmgcl 3-hydroxy-3-methylglutaryl-Coenzyme A lyase

Hmgcll1 3-hydroxymethyl-3-methylglutaryl-Coenzyme A

Hn1 hematological and neurological expressed

Hn1l hematological and neurological expressed 1-like

Hnrnpc heterogeneous nuclear ribonucleoprotein C

Hnrnpf heterogeneous nuclear ribonucleoprotein $\mathrm{F}$

Hnrpdl heterogeneous nuclear ribonucleoprotein D-like

Hook1 hook homolog 1

Hormad1 HORMA domain containing 1 
Hoxa11 homeobox A11

Hoxb4 homeo box B4

Hoxd1 homeo box D1

Hoxd3 homeo box D3

$\mathrm{Hr}$ hairless protein

Hs1bp3 HCLS1 binding protein 3

Hs2st1 heparan sulfate 2-O-sulfotransferase 1

Hsd11b1 hydroxysteroid 11-beta dehydrogenase 1

Hsd17b2 hydroxysteroid (17-beta) dehydrogenase 2

Hsd3b2 hydroxy-delta-5-steroid dehydrogenase 3 beta-

Hsd3b6 hydroxy-delta-5-steroid dehydrogenase 3 beta-

Hspb2 heat shock protein 2

Htr4 serotonin 5-HT4 receptor

Htr5a 5-hydroxytryptamine receptor 5A

lars isoleucyl-tRNA synthetase

Idh2 isocitrate dehydrogenase 2 (NADP+)

Idua iduronidase alpha-L-

Igdcc3 putative neuronal cell adhesion molecule

Igdcc4 neighbor of Punc e11 protein

Igf1r insulin-like growth factor I receptor

Igf2bp1 insulin-like growth factor 2 mRNA binding

Igf2bp2 insulin-like growth factor 2 mRNA binding

Igf2bp3 insulin-like growth factor 2 mRNA binding

Igf2r insulin-like growth factor 2 receptor

Igfbp5 insulin-like growth factor binding protein 5

Igsf1 immunoglobulin superfamily member 1 long

Igsf9 immunoglobulin superfamily member 9

Ikbkap inhibitor of kappa light polypeptide enhancer in

Ikbke inhibitor of kappaB kinase epsilon

Ikzf2 helios

Ikzf4 zinc finger protein subfamily 1A 4

Il10 interleukin 10

Il13 interleukin 13

II17rd interleukin 17 receptor D

Il1a interleukin 1 alpha

II20rb interleukin 20 receptor beta precursor

II28ra interleukin 28 receptor alpha

II6 interleukin 6

Imp3 IMP3 U3 small nucleolar ribonucleoprotein 
Impg2 interphotoreceptor matrix proteoglycan 2

Ing3 inhibitor of growth family member 3

Ino80d hypothetical protein LOC227195 isoform 2

Insr insulin receptor

Ints12 integrator complex subunit 12

Ints2 integrator complex subunit 2

Intu PDZ domain containing 6

Ip6k2 inositol hexaphosphate kinase 2

Ipo11 importin 11

Ipo9 importin 9

Iqcb1 IQ calmodulin-binding motif containing 1

Iqsec2 IQ motif and Sec7 domain 2 isoform 2

Iqsec3 IQ motif and Sec7 domain 3

Irf4 interferon regulatory factor 4

Irs1 insulin receptor substrate 1

IsI1 ISL1 transcription factor LIM/homeodomain

Itga4 integrin alpha 4

Itga8 integrin alpha 8

Itgav integrin alpha $\mathrm{V}$

Itgb3 integrin beta 3 precursor

Itpkc inositol 145-trisphosphate 3-kinase C

Itsn 1 intersectin 1 isoform 1

Jam3 junction adhesion molecule 3

Kank4 KN motif and ankyrin repeat domains 4

Kbtbd13 hypothetical protein LOC74492

Kcna1 potassium voltage-gated channel subfamily $A$

Kcna4 potassium voltage-gated channel shaker-related

Kcna6 potassium voltage-gated channel shaker-related

Kcnab1 potassium voltage-gated channel shaker-related

Kcnc1 Shaw-related voltage-gated potassium channel

Kcnc2 potassium voltage gated channel Shaw-related

Kcnc3 potassium voltage gated channel Shaw-related

Kcnc4 potassium voltage gated channel Shaw-related

Kcnd3 potassium voltage-gated channel Shal-related

Kcnj6 potassium inwardly-rectifying channel J6 isoform

Kcnk18 2P K ion channel TRESK

Kcnk3 potassium channel subfamily $\mathrm{K}$ member 3

Kctd12b potassium channel tetramerisation domain

Kctd17 potassium channel tetramerisation domain 
Kctd21 potassium channel tetramerisation domain

Kctd7 potassium channel tetramerisation domain

Kdelc2 KDEL (Lys-Asp-Glu-Leu) containing 2 protein

$\mathrm{Kdm} 4 \mathrm{~d}$ jumonji domain containing 2D

Khsrp KH-type splicing regulatory protein (FUSE

Kif11 kinesin family member 11

Kif14 kinesin family member 14

Kif16b kinesin family member 16B

Kif1b kinesin family member $1 \mathrm{~B}$ isoform a

Kif21b kinesin family member 21B

Kif2a kinesin family member $2 \mathrm{~A}$

Kif2b kinesin family member $2 \mathrm{~B}$

Kif3a kinesin family member $3 \mathrm{~A}$

Kifc2 kinesin family member C2

KIf8 Kruppel-like factor 8

KIhdc7a kelch domain containing 7A

KIhdc8b kelch domain containing $8 B$

KIhl13 kelch-like 13

KIhl18 kelch-like 18

KIhl23 kelch-like 23

KIhl24 kelch-like 24

KIhl30 kelch-like 30

KIhl31 kelch repeat and BTB (POZ) domain containing 1

KIhl4 kelch-like 4

KIhl6 kelch-like 6

KIhl9 kelch-like 9

KIk10 kallikrein related-peptidase 10

Klkb1 kallikrein B plasma 1

KIrg2 killer cell lectin-like receptor subfamily G

Kpna1 karyopherin (importin) alpha 1

Kpna4 karyopherin alpha 4

Kremen 1 kringle-containing transmembrane protein 1

Kremen2 kringle-containing transmembrane protein 2

Krt26 keratin 26

Krt6a keratin 6A

Krt6b keratin complex 2 basic gene $6 b$

Lactb lactamase beta

Lalba lactalbumin alpha

Lama1 laminin alpha 1 precursor 
Larp4 La ribonucleoprotein domain family member 4

Lars2 leucyl-tRNA synthetase mitochondrial

Lbh limb-bud and heart

Lbr lamin B receptor

Lcor ligand dependent nuclear receptor corepressor

Lcorl ligand dependent nuclear receptor

Ldb3 LIM domain binding 3 isoform $b$

Ldoc1l leucine zipper down-regulated in cancer 1-like

Leprotl1 leptin receptor overlapping transcript-like 1

Letmd1 LETM1 domain containing 1

Lgr4 G protein-coupled receptor 48

Lhx9 LIM homeobox protein 9 isoform b

Lifr leukemia inhibitory factor receptor isoform 2

Limd1 LIM domains containing 1

Limd2 LIM domain containing 2

Lin28a RNA-binding protein LIN-28

Lin28b lin-28 homolog $b$

Lingo1 leucine rich repeat and Ig domain containing 1

Lman1 lectin mannose-binding 1

Lmln leishmanolysin-like (metallopeptidase M8

Lmod1 leiomodin 1 (smooth muscle)

Lmtk2 lemur tyrosine kinase 2

Lmx1a LIM homeobox transcription factor 1 alpha

Lonrf3 ring finger protein 127

Lor loricrin

Lox lysyl oxidase

Loxl3 lysyl oxidase-like 3

Lpar2 endothelial differentiation lysophosphatidic

Lpgat1 lysophosphatidylglycerol acyltransferase 1

Lphn1 latrophilin 1 precursor

Lrdd leucine-rich and death domain containing

Lrfn4 leucine rich repeat and fibronectin type III

Lrig1 leucine-rich repeats and immunoglobulin-like

Lrig2 leucine-rich repeats and immunoglobulin-like

Lrig3 leucine-rich repeats and immunoglobulin-like

Lrp11 low density lipoprotein receptor-related protein

Lrp2bp LRP2 binding protein

Lrrc15 leucine rich repeat containing 15

Lrrc2 leucine rich repeat containing 2 
Lrrc20 leucine rich repeat containing 20

Lrrc4 leucine rich repeat containing 4

Lrrc41 leucine rich repeat containing 41

Lrrc58 leucine rich repeat containing 58

Lrrc59 leucine rich repeat containing 59

Lrtm2 leucine-rich repeats and transmembrane domains

Lsm11 U7 snRNA-associated Sm-like protein Lsm11

Luc7|3 cisplatin resistance-associated overexpressed

Ly6g6c lymphocyte antigen 6 complex G6C

Lynx1 Ly6/neurotoxin 1

Lysmd3 LysM putative peptidoglycan-binding domain

Lyve1 lymphatic vessel endothelial hyaluronan receptor

Magt1 implantation-associated protein

Mamdc2 MAM domain containing 2

Man1a2 mannosidase alpha class $1 \mathrm{~A}$ member 2

Man2a1 mannosidase 2 alpha 1

Maneal hypothetical protein LOC215090

Map3k1 mitogen-activated protein kinase kinase kinase

Map3k13 mitogen-activated protein kinase kinase kinase

Map3k2 mitogen activated protein kinase kinase kinase

Map3k3 mitogen-activated protein kinase kinase kinase

Map4k3 mitogen-activated protein kinase kinase kinase

Mapk11 mitogen-activated protein kinase 11

Mapk9 mitogen activated protein kinase 9 isoform b

Mapre1 microtubule-associated protein RP/EB family

MarcksI1 MARCKS-like 1

Mars2 mitochondrial methionyl-tRNA synthetase

Marveld2 MARVEL (membrane-associating) domain containing

Masp1 mannan-binding lectin serine protease 1

Mbnl3 muscleblind-like 3

Mbtd1 mbt domain containing 1

Mbtps2 membrane-bound transcription factor peptidase

Mcfd2 multiple coagulation factor deficiency 2

Mctp1 multiple C2 domains transmembrane 1

Mctp2 multiple C2 domains transmembrane 2

Mcts2 malignant T cell amplified sequence 2

Mdc1 mediator of DNA damage checkpoint 1

Me3 malic enzyme 3 NADP(+)-dependent

Med13 mediator complex subunit 13 
Med13I thyroid hormone receptor associated protein 2

Med28 endothelial-derived gene 1

Med6 mediator of RNA polymerase II transcription

Med8 mediator of RNA polymerase II transcription

Meis3 myeloid ecotropic viral integration site-related

Mesdc1 mesoderm development candidate 1

Mex3a mex3 homolog A

Mfsd4 major facilitator superfamily domain containing

Mga MAX-interacting protein

Mgat4a mannoside acetylglucosaminyltransferase 4

Midn midnolin

Mier1 mesoderm induction early response 1 isoform $b$

Mier3 mesoderm induction early response 1 family

Mlst8 $\mathrm{G}$ protein beta subunit-like

MIxip MLX interacting protein isoform 1

Mmd monocyte to macrophage

Mmgt1 transmembrane protein 32

Mmp11 matrix metallopeptidase 11

Mmp1a matrix metalloproteinase 1a (interstitial

Mmp1b matrix metalloproteinase $1 b$ precursor

Mnt max binding protein

Mpa2I macrophage activation 2 like

Mpdz multiple PDZ domain protein

Mpi mannose phosphate isomerase

Mpp2 membrane protein palmitoylated 2 (MAGUK p55

Mri1 hypothetical protein LOC67873

Mrpl21 mitochondrial ribosomal protein L21

Ms4a13 membrane-spanning 4-domains subfamily A member

Msi2 Musashi homolog 2

Msn moesin

Mtch2 mitochondrial carrier homolog 2

Mterfd3 transcription termination factor-like protein

Mtf1 metal response element binding transcription

Mtf2 metal response element binding transcription

Mtmr14 myotubularin related protein 14

Mtmr4 myotubularin related protein 4

Mtor FK506 binding protein 12-rapamycin associated

Mtpn myotrophin

Mxd1 MAX dimerization protein 1 
Mycbp c-myc binding protein

Mycl1 lung carcinoma myc related oncogene 1

Mycn neuroblastoma myc-related oncogene 1

Myh10 myosin heavy chain 10 non-muscle

Myo1f myosin IF

Myo5a myosin Va

Myo7a myosin VIla

Myrip myosin VIIA and Rab interacting protein

Myst3 MYST histone acetyltransferase (monocytic

Naa15 NMDA receptor-regulated gene 1

Naa30 N-acetyltransferase 12

Nacc1 transcriptional repressor NAC1

Nap1l1 nucleosome assembly protein 1-like 1

Napepld N-acyl phosphatidylethanolamine phospholipase D

Nars2 asparaginyl-tRNA synthetase 2

Nat8I N-acetyltransferase 8-like

Nceh1 arylacetamide deacetylase-like 1

Nckap1l NCK associated protein 1 like

Ncoa3 nuclear receptor coactivator 3

Ncor1 nuclear receptor co-repressor 1

Ndrg2 N-myc downstream regulated gene 2

Ndst2 N-deacetylase/N-sulfotransferase (heparan

Ndst3 N-deacetylase/N-sulfotransferase (heparan

Necap1 NECAP endocytosis associated 1

Nedd4I neural precursor cell expressed developmentally

Nefm neurofilament 3 medium

Nek9 NIMA-related expressed kinase 9

Neu3 neuraminidase 3

Neurog3 neurogenin 3

$\mathrm{Nf} 2$ neurofibromatosis 2

Nfam1 NFAT activation molecule 1 protein

Nfia nuclear factor I/A isoform 2

Nfib nuclear factor I/B isoform 2

Nfxl1 ovarian zinc finger protein

Ngf nerve growth factor beta isoform B

Ngly1 N-glycanase 1

Nhedc2 $\mathrm{Na}+/ \mathrm{H}+$ exchanger domain containing 2

Nid1 nidogen 1

Nid2 nidogen 2 
Nin ninein isoform 1

Nipa2 non-imprinted in Prader-Willi/Angelman syndrome

Nipal1 NIPA-like domain containing 1

Nipal4 hypothetical protein LOC214112

Nipsnap1 4-nitrophenylphosphatase domain and non-neuronal

Nkain1 $\mathrm{Na}+/ \mathrm{K}+$ transporting ATPase interacting 1

Nkap NFKB activating protein

Nkiras2 NFKB inhibitor interacting Ras-like protein 2

Nlk nemo like kinase

NIrp4c NLR family pyrin domain containing 4C

Nme4 nucleoside diphosphate kinase 4

Nme6 non-metastatic cells 6 protein expressed in

Nmnat2 nicotinamide nucleotide adenylyltransferase 2

Notch2 Notch gene homolog 2

Npc2 epididymal secretory protein E1

Npepl1 aminopeptidase-like 1

Nphp3 nephrocystin 3 isoform b

Nqo1 NAD(P)H dehydrogenase quinone 1

Nr1d2 nuclear receptor subfamily 1 group D member 2

Nr6a1 nuclear receptor subfamily 6 group A member 1

Nrarp Notch-regulated ankyrin repeat protein

Nras neuroblastoma ras oncogene

Nrtn neurturin

Nsd1 nuclear receptor-binding SET-domain protein 1

Nsmce2 non-SMC element 2 MMS21 homolog

Nsun2 NOL1/NOP2/Sun domain family 2

Nt5e 5 ' nucleotidase ecto

Nt5m 5'3'-nucleotidase mitochondrial

Ntn1 netrin 1

Nuak1 NUAK family SNF1-like kinase 1

Numbl numb-like

Nup155 nucleoporin 155

Nup214 nucleoporin 214

Nxt2 nuclear transport factor 2-like export factor 2

Obfc2a oligonucleotide/oligosaccharide-binding fold

Ocrl phosphatidylinositol polyphosphate

Odz2 odd Oz/ten-m homolog 2 isoform 1

Ogfr opioid growth factor receptor

Olfr121 olfactory receptor 121 
Olr1 oxidized low density lipoprotein (lectin-like)

Onecut2 one cut domain family member 2

Onecut3 one cut domain family member 3

Osbp2 oxysterol binding protein 2

Osmr oncostatin $\mathrm{M}$ receptor

Ostf1 osteoclast stimulating factor 1

Otud7b zinc finger A20 domain containing 1

Oxa1l oxidase assembly 1-like

P2rx1 purinergic receptor $\mathrm{P} 2 \mathrm{X} 1$

P2ry4 pyrimidinergic receptor P2Y G-protein coupled

P4ha2 procollagen-proline 2-oxoglutarate

Pa2g4 ErbB3-binding protein 1

Pabpc4I poly $(A)$ binding protein cytoplasmic 4-like

Pacs2 phosphofurin acidic cluster sorting protein 2

Pak1 p21 (CDKN1A)-activated kinase 1

Panx2 pannexin 2

Pappa pregnancy-associated plasma protein $A$

Parp14 poly (ADP-ribose) polymerase family member 14

Parp16 poly (ADP-ribose) polymerase family member 16

Pars2 prolyl-tRNA synthetase (mitochondrial)(putative)

Patl1 protein associated with topoisomerase II homolog

Pbx1 pre B-cell leukemia transcription factor 1

Pbx2 pre B-cell leukemia transcription factor 2

Pbx3 pre B-cell leukemia transcription factor 3

Pcdh19 protocadherin 19 isoform b

Pcdh20 protocadherin 20

Pcdh7 protocadherin 7 isoform 1

Pcgf3 polycomb group ring finger 3

Pck2 mitochondrial phosphoenolpyruvate carboxykinase

Pcp4l1 Purkinje cell protein 4-like 1

Pctp phosphatidylcholine transfer protein

Pcyt1b phosphate cytidylyltransferase 1 choline beta

Pdcd5 programmed cell death 5

Pdcd7 programmed cell death protein 7

Pde10a phosphodiesterase 10A

Pde12 2'-phosphodiesterase

Pde1c phosphodiesterase $1 \mathrm{C}$ isoform a

Pdgfb platelet derived growth factor B polypeptide

Pdp2 pyruvate dehydrogenase phosphatase isoenzyme 2 
Pdpk1 3-phosphoinositide dependent protein kinase-1

Pdxk pyridoxal (pyridoxine vitamin B6) kinase

Pdzd3 PDZ domain containing 3

Peg3 paternally expressed 3

Penk preproenkephalin 1

Per3 period homolog 3

Pex26 peroxisome biogenesis factor 26

Pfkfb1 6-phosphofructo-2-kinase/fructose-2

Pgam1 phosphoglycerate mutase 1

Pgm2l1 phosphoglucomutase 2-like 1

Pgm3 phosphoglucomutase 3

Pgr15I G protein-coupled receptor 15-like

Pgrmc1 progesterone receptor membrane component

Phf21a PHD finger protein 21A isoform 2

Phf8 PHD finger protein 8 isoform a

Phyhip phytanoyl-CoA hydroxylase interacting protein

Pi4k2b phosphatidylinositol 4-kinase type 2 beta

Pias4 protein inhibitor of activated STAT 4

Piga phosphatidylinositol glycan class A

Pigm phosphatidylinositol glycan anchor biosynthesis

Pigw phosphatidylinositol glycan class W

Pik3ip1 calcineurin-regulated kringle domain protein

Pikfyve 1-phosphatidylinositol-4-phosphate 5-kinase

Pim1 proviral integration site 1

Pip4k2b phosphatidylinositol-5-phosphate 4-kinase type

Pkd1 polycystin 1

Pkp1 plakophilin 1

Pla2g15 lysophospholipase 3

Pla2g2f phospholipase A2 group IIF

Pla2g3 phospholipase A2 group III

Plaa phospholipase A2 activating protein

Plagl2 pleiomorphic adenoma gene-like 2

PId3 phospholipase D family member 3

PId5 phospholipase D family member 5

PId6 hypothetical protein LOC194908

PIdn pallidin

Plekhb2 pleckstrin homology domain containing family $B$

Plekhg6 pleckstrin homology domain containing family $G$

Plekhj1 guanine nucleotide releasing protein $\mathrm{x}$ 
Plekho1 TNF intracellular domain-interacting protein

PIp1 proteolipid protein 1

Plxna2 plexin A2

PIxna4 plexin A4

Plxnc1 plexin C1

Plxnd1 plexin D1

Pmaip1 phorbol-12-myristate-13-acetate-induced protein

Pnp purine-nucleoside phosphorylase

Pofut1 protein O-fucosyltransferase 1 isoform 2

Pogz pogo transposable element with ZNF domain

Pole4 DNA polymerase epsilon subunit 4

Polr3d polymerase (RNA) III (DNA directed) polypeptide

Polr3g polymerase (RNA) III (DNA directed) polypeptide

Pom121 nuclear pore membrane protein 121

Ppapdc1b phosphatidic acid phosphatase type 2 domain

Ppapdc2 phosphatidic acid phosphatase type 2 domain

Ppargcla peroxisome proliferative activated receptor

Ppargc1b peroxisome proliferative activated receptor

Ppm1f protein phosphatase 1F (PP2C domain containing)

Ppme1 protein phosphatase methylesterase 1

Ppp1r2 protein phosphatase 1 regulatory (inhibitor)

Ppp1r3a protein phosphatase 1 regulatory (inhibitor)

Ppp2r1b protein phosphatase 2 (formerly 2A) regulatory

Ppp3r1 protein phosphatase 3 regulatory subunit $B$

Ppt2 palmitoyl-protein thioesterase 2

Pptc7 T-cell activation protein phosphatase 2C

Pqlc2 PQ loop repeat containing 2

Prdm1 PR domain containing 1 with ZNF domain

Prickle1 prickle like 1

Prkab2 AMP-activated protein kinase beta 2

Prkx protein kinase $\mathrm{X}$-linked

Prmt6 HMT1 hnRNP methyltransferase-like 6

Prodh proline dehydrogenase

Prpf18 PRP18 pre-mRNA processing factor 18 homolog

Prpf38b PRP38 pre-mRNA processing factor 38 (yeast)

Prpf39 PRP39 pre-mRNA processing factor 39 homolog

Prps2 phosphoribosyl pyrophosphate synthetase 2

Prr18 proline rich region 18

Prr3 proline-rich protein 3 
Prr5I hypothetical protein LOC72446

Prr9 hypothetical protein LOC109314

Prrg3 proline rich Gla (G-carboxyglutamic acid) 3

Prrx1 paired related homeobox 1 isoform a

Prss22 protease serine 22

Prss53 hypothetical protein LOC330657

Prtg protogenin

Psd3 pleckstrin and Sec7 domain containing 3 isoform

Psg17 pregnancy specific glycoprotein 17

Pskh1 protein serine kinase $\mathrm{H} 1$

Psme3 proteasome activator subunit 3

Psors1c2 MHC psoriasis candidate

Pten phosphatase and tensin homolog

Ptgs2 prostaglandin-endoperoxide synthase 2

Ptk7 PTK7 protein tyrosine kinase 7

Ptprd protein tyrosine phosphatase receptor type D

Ptprg protein tyrosine phosphatase receptor type G

Ptpru protein tyrosine phosphatase receptor type $U$

Purg purine-rich element binding protein $\mathrm{G}$ isoform a

Pvr poliovirus receptor

Pygo2 pygopus 2

Qars glutaminyl-tRNA synthetase

Rab11fip1 RAB11 family interacting protein 1

Rab11fip4 Rab11-FIP4-like

Rab15 RAB15 member RAS oncogene family

Rab40c Rab40c member RAS oncogene family

Rab6b RAB6B member RAS oncogene family

Rab8b RAB8B member RAS oncogene family

Rabgap1l RAB GTPase activating protein 1-like isoform b

Rabgef1 RAB guanine nucleotide exchange factor (GEF) 1

Rabl3 RAB member of RAS oncogene family-like 3

Rad23b RAD23b homolog

Rad51I3 RAD51-like 3

Ralb v-ral simian leukemia viral oncogene homolog B

Ralgds ral guanine nucleotide dissociation stimulator

Ramp1 receptor-activity modifying protein 1 isoform 2

Ranbp10 Ran-binding protein 10

Rap1gap2 GTPase activating RANGAP domain-like 4

Rapgefl1 Rap guanine nucleotide exchange factor 
Rarg retinoic acid receptor gamma isoform 2

Rasgrp1 RAS guanyl releasing protein 1

Rasl10a RAS-related on chromosome 22

Rasl10b RAS-like family 10 member B

Rassf3 Ras association (RalGDS/AF-6) domain family

Raver2 raver2

Rb1 retinoblastoma 1

Rbm14 RNA binding motif protein 14

Rbm19 RNA binding motif protein 19

Rbm38 seb4

Rbm4 RNA binding motif protein 4

Rbms2 RNA binding motif single stranded interacting

$\mathrm{Rbpj}$ recombining binding protein suppressor of

Rcn1 reticulocalbin 1

Rdh10 retinol dehydrogenase 10

Rdh12 retinol dehydrogenase 12

Rdh13 retinol dehydrogenase 13 (all-trans and 9-cis)

$\mathrm{Rdx}$ radixin isoform a

Reck reversion-inducing-cysteine-rich protein with

Reep1 receptor accessory protein 1

Reep4 receptor accessory protein 4

Rfx5 regulatory factor $X 5$

Rg9mtd2 RNA (guanine-9-) methyltransferase domain

Rgp1 RGP1 retrograde golgi transport homolog

Rgr retinal G protein coupled receptor

Rgs16 regulator of G-protein signaling 16

Rgs8 regulator of $\mathrm{G}$-protein signalling 8

Rgs9bp regulator of $G$ protein signaling 9-binding

Rhbdd2 rhomboid domain containing 2

Rhof ras homolog gene family member $f$

Rhoh ras homolog gene family member $\mathrm{H}$

Rhoj ras homolog gene family member $\mathrm{J}$

Rictor RIKEN cDNA 4921505C17

Rimkla hypothetical protein LOC194237

Rimklb hypothetical protein LOC108653

Rinl hypothetical protein LOC320435

Riok3 RIO kinase 3

Rlim ring finger protein 12

Rmi1 RMI1 RecQ mediated genome instability 1 
Rnf115 Rabring 7

Rnf13 ring finger protein 13 isoform a

Rnf135 ring finger protein 135

Rnf139 ring finger protein 139

Rnf170 ring finger protein 170

Rnf222 hypothetical protein LOC320040

Rnf44 ring finger protein 44

Rnf5 ring finger protein 5

Rnf7 ring finger protein 7

Rnf8 ring finger protein 8

Rorc RAR-related orphan receptor gamma

Rpgrip1l Rpgrip1-like

Rprd2 hypothetical protein LOC75137

Rpusd2 RNA pseudouridylate synthase domain containing

Rragc small GTPase homolog

Rragd Ras-related GTP binding D

Rrm2 ribonucleotide reductase $\mathrm{M} 2$

Rrp12 ribosomal RNA processing 12 homolog

Rsad2 radical S-adenosyl methionine domain containing

Rspo2 R-spondin 2 homolog

Rspry1 ring finger and SPRY domain containing 1

Rtcd1 RNA terminal phosphate cyclase domain 1

Rtp1 receptor transporter protein 1

Rufy2 RUN and FYVE domain-containing 2

Rufy3 RUN and FYVE domain containing 3

Runx1t1 acute myelogenous leukemia 1 translocation 1

Sall3 sal-like protein 3

Sall4 sal-like 4 isoform b

Samd8 sterile alpha motif domain containing 8

Sart1 squamous cell carcinoma antigen recognized by

Sass 6 spindle assembly 6

Sbk1 SH3-binding kinase 1

Sbno1 sno strawberry notch homolog 1

Scai hypothetical protein LOC320271

Scara3 scavenger receptor class A member 3

Scd1 stearoyl-Coenzyme A desaturase 1

Scd2 stearoyl-Coenzyme A desaturase 2

Scml2 sex comb on midleg-like 2

Scn11a sodium channel voltage-gated type XI alpha 
Scn4b sodium channel type IV beta

Scn5a voltage-gated sodium channel type $V$ alpha

Scn8a sodium channel voltage-gated type VIII alpha

Scoc short coiled-coil protein isoform a

Scyl3 ezrin-binding partner PACE-1

Sdad1 SDA1 domain containing 1

Sdc2 syndecan 2

Sdhaf2 hypothetical protein LOC66072

Sdk1 sidekick 1

Sec14I1 SEC14-like 1

Sec16b SEC16 homolog B

Sec22a SEC22 vesicle trafficking protein homolog $A$

Sec24c SEC24 related gene family member C

Sec63 SEC63-like protein

Sectm1a secreted and transmembrane $1 \mathrm{~A}$

Sel1/3 hypothetical protein LOC231238

Sema3f sema domain immunoglobulin domain (Ig) short

Sema3g sema domain immunoglobulin domain (Ig) short

Sema4f sema domain immunoglobulin domain (Ig) TM

Sema4g semaphorin $4 G$

Senp2 SUMO/sentrin specific peptidase 2

Senp5 SUMO/sentrin specific peptidase 5

Serac1 serine active site containing 1 isoform 2

Serbp1 SERPINE1 mRNA binding protein 1 isoform 4

Serf2 small EDRK-rich factor 2

Serinc4 serine incorporator 4

Setd5 SET domain containing 5

Sf1 splicing factor 1 isoform 2

Sf3a1 splicing factor 3a subunit 1

Sfxn 5 sideroflexin 5

Sgcb sarcoglycan beta (dystrophin-associated

Sgk2 serum/glucocorticoid regulated kinase 2

Sgms2 sphingomyelin synthase 2

Sgpp2 sphingosine-1-phosphate phosphotase 2

Sh2b3 SH2B adaptor protein 3

Sh2d1b1 SH2 domain protein 1B

Sh3bgrl2 SH3 domain binding glutamic acid-rich protein

Sh3d19 SH3 domain protein D19

Sh3rf3 SH3 multiple domains 4 
Shc1 src homology 2 domain-containing transforming

Shprh SNF2 histone linker PHD RING helicase isoform $b$

Siglec1 sialoadhesin

Sirt7 sirtuin 7 (silent mating type information

Sit1 SHP2-interacting transmembrane adaptor protein

Sla src-like adaptor isoform b

Slamf1 signaling lymphocytic activation molecule family

Slamf6 SLAM family member 6

SIc10a6 sodium-dependent organic anion transporter

Slc10a7 solute carrier family 10 (sodium/bile acid

SIc12a9 solute carrier family 12 (potassium/chloride

SIc13a4 solute carrier family 13 (sodium/sulfate

Slc15a1 solute carrier family 15 (oligopeptide

Slc15a2 solute carrier family $15(\mathrm{H}+/$ peptide

SIc16a14 solute carrier family 16 (monocarboxylic acid

Slc16a6 solute carrier family 16 member 6 isoform b

Slc16a9 solute carrier family 16 (monocarboxylic acid

SIc17a9 hypothetical protein LOC228993

Slc20a1 solute carrier family 20 member 1

Slc22a23 solute carrier family 22 member 23

Slc23a2 solute carrier family 23 (nucleobase

Slc24a2 solute carrier family 24

SIc24a3 solute carrier family 24

Slc25a16 solute carrier family 25 (mitochondrial carrier SIc25a18 solute carrier family 25 (mitochondrial

SIc25a22 solute carrier family 25 (mitochondrial carrier

Slc25a23 solute carrier family 25 member 23

SIc25a24 calcium-binding transporter

SIc25a27 solute carrier family 25 member 27

SIc25a4 solute carrier family 25 (mitochondrial carrier

Slc25a45 solute carrier family 25 member 45

Slc26a1 solute carrier family 26 (sulfate transporter)

Slc26a5 prestin

Slc26a6 solute carrier family 26 member 6

SIc26a9 solute carrier family 26 member 9

Slc28a1 solute carrier family 28 (sodium-coupled

Slc29a2 solute carrier family 29 (nucleoside

SIc2a3 solute carrier family 2 (facilitated glucose

Slc2a6 solute carrier family 2 (facilitated glucose 
SIc2a8 solute carrier family 2 (facilitated glucose

Slc30a1 solute carrier family 30 (zinc transporter)

SIc30a4 solute carrier family 30 (zinc transporter)

SIc31a1 solute carrier family 31 member 1

Slc31a2 solute carrier family 31 member 2

Slc35b4 solute carrier family 35 member B4

SIc35c1 GDP-fucose transporter 1 isoform 2

Slc35d2 solute carrier family 35 member D2

Slc36a1 solute carrier family 36 member 1

Slc37a4 solute carrier family 37 (glucose-6-phosphate

SIc38a8 solute carrier family 38 member 8

Slc38a9 solute carrier family 38 member 9

Slc41a2 solute carrier family 41 member 2

Slc4a4 solute carrier family 4 (anion exchanger)

Slc4a7 solute carrier family 4 sodium bicarbonate

Slc5a9 solute carrier family 5 (sodium/glucose

SIc6a1 solute carrier family 6 (neurotransmitter

SIc6a15 solute carrier family 6 member 15

SIc6a6 solute carrier family 6 (neurotransmitter

Slc7a11 solute carrier family 7 (cationic amino acid

Slc7a14 solute carrier family 7 member 14

Slc8a2 solute carrier family 8 (sodium/calcium

SIc9a4 solute carrier family 9 (sodium/hydrogen

Slco2a1 solute carrier organic anion transporter family

Slco5a1 solute carrier organic anion transporter family

SIfn1 schlafen 1

Slitrk4 SLIT and NTRK-like family member 4

SImap sarcolemma associated protein

Smap1 stromal membrane-associated protein 1

Smap2 stromal membrane-associated GTPase-activating

Smarcad1 SWI/SNF-related matrix-associated

Smarcc1 SWI/SNF related matrix associated actin

Smarcd1 SWI/SNF related matrix associated actin

Smcr7 Smith-Magenis syndrome chromosome region

Smcr7l hypothetical protein LOC239555

Smcr8 Smith-Magenis syndrome chromosome region

Smug1 single-strand selective monofunctional uracil

Smyd5 SET and MYND domain containing 5

Snai1 snail homolog 1 
Snai3 snail homolog 3

Snap23 synaptosomal-associated protein 23

Snap29 GS32 protein

Snap91 synaptosomal-associated protein 91

Snn stannin

Snph syntaphilin

Sntn hypothetical protein LOC218739

Snx16 sorting nexin 16

Snx30 sorting nexin family member 30

Soat2 acyl-CoA:cholesterol acyltransferase 2

Socs1 suppressor of cytokine signaling 1

Socs7 suppressor of cytokine signaling 7

Sod2 superoxide dismutase 2 mitochondrial

Sorcs1 SORCS receptor 1

Sort1 sortilin 1

Sox13 SRY-box 13

Sp8 trans-acting transcription factor 8

Spata2 spermatogenesis associated 2

Spata 6 spermatogenesis associated 6

Spats2I hypothetical protein LOC67198

Spcs3 signal peptidase complex subunit 3

Spin1 spindlin isoform 2

Spink5 serine peptidase inhibitor Kazal type 5

Spint1 Kunitz-type protease inhibitor 1 precursor

Spnb2 spectrin beta 2 isoform 2

Spnb4 spectrin beta 4

Spon2 spondin 2 extracellular matrix protein

Spred3 sprouty-related EVH1 domain containing 3

Sprn shadow of prion protein

Spryd4 SPRY domain containing 4

Spsb4 splA/ryanodine receptor domain and SOCS box

Sptlc2 serine palmitoyltransferase long chain base

Spty2d1 SPT2 Suppressor of Ty domain containing 1

Srebf2 sterol regulatory element binding factor 2

Srgap3 SLIT-ROBO Rho GTPase activating protein 3

Srpk2 serine/arginine-rich protein specific kinase 2

Srpr signal recognition particle receptor

Srr serine racemase

Srrm4 hypothetical protein LOC68955 
Ss18I1 synovial sarcoma translocation gene on

Ssr1 signal sequence receptor alpha

Sstr3 somatostatin receptor 3

Ssx2ip synovial sarcoma $X$ breakpoint 2 interacting

St13 suppression of tumorigenicity 13

St3gal1 ST3 beta-galactoside alpha-23-sialyltransferase

St6gal1 beta galactoside alpha 26 sialyltransferase 1

St8sia1 ST8 alpha-N-acetyl-neuraminide

Stab2 stabilin-2

Stard13 StAR-related lipid transfer (START) domain

Stard3nl MLN64 N-terminal homolog

Stat2 signal transducer and activator of transcription

Stim1 stromal interaction molecule 1

Stk24 serine/threonine protein kinase 24

Stk35 serine/threonine kinase 35 isoform b

Ston2 stonin 2

Stox2 storkhead box 2 isoform 1

Strbp spermatid perinuclear RNA-binding protein

Stx17 syntaxin 17

Stx3 syntaxin 3 isoform B

Stxbp5 tomosyn

Styk1 serine/threonine/tyrosine kinase 1

Styx phosphoserine/threonine/tyrosine interaction

Sub1 RNA polymerase II transcriptional coactivator

Sumf2 sulfatase modifying factor 2

Supt6h suppressor of Ty 6 homolog

Surf4 surfeit gene 4

Susd2 sushi domain containing 2

Suv39h1 suppressor of variegation 3-9 homolog 1

Suv39h2 suppressor of variegation 3-9 homologue 2

Syn2 synapsin II isoform Ila

Syncrip NS1-associated protein 1 isoform 1

Synj2bp synaptojanin 2 binding protein

Syt11 synaptotagmin XI

Syt2 synaptotagmin II

Sytl4 synaptotagmin-like 4

Tab2 mitogen-activated protein kinase kinase kinase 7

Tac4 tachykinin 4

Taf5 Tbp-associated factor 5 
Taf9b TAF9-like RNA polymerase II TATA box binding

Taok1 TAO kinase 1

Tarbp2 TAR (HIV) RNA binding protein 2

Tat tyrosine aminotransferase

Tbc1d13 TBC1 domain family member 13

Tbkbp1 ProSAPiP2 protein

Tbx19 T-box 19

Tbx5 T-box 5

Tcta T-cell leukemia translocation altered

Tcte1 t-complex-associated testis expressed 1

Tdgf1 teratocarcinoma-derived growth factor

Tdrkh tudor and $\mathrm{KH}$ domain containing protein

Tead4 TEA domain family member 4 isoform b

Tecpr2 hypothetical protein LOC104859

Tef thyrotroph embryonic factor isoform 2

Tes testis derived transcript

Tet2 tet oncogene family member 2

Tfdp2 transcription factor Dp-2

Tgds TDP-glucose 46-dehydratase

Tgfbr1 transforming growth factor beta receptor I

Tgfbr3 transforming growth factor beta receptor III

Tgif2 TGFB-induced factor 2

Tgs1 nuclear receptor coactivator 6 interacting

Thbd thrombomodulin

Thbs1 thrombospondin 1

Thoc1 THO complex 1

Thoc2 THO complex subunit 2

Thrsp thyroid hormone-responsive protein

Tia1 cytotoxic granule-associated RNA binding protein

Timm17b translocase of inner mitochondrial membrane $17 \mathrm{~b}$

Timm8a1 translocase of inner mitochondrial membrane 8

Tirap Toll-interleukin 1 receptor domain-containing

Tjp1 tight junction protein 1

Tle4 transducin-like enhancer protein 4

TIk2 tousled-like kinase 2 isoform A

TIn1 talin 1

TIn2 talin 2

Tm4sf5 transmembrane 4 superfamily member 5

Tm6sf1 transmembrane 6 superfamily member 1 
Tmc7 Tmc7 protein

Tmed10 transmembrane emp24 domain-containing protein

Tmed4 transmembrane emp24 protein transport domain

Tmed5 transmembrane emp24 protein transport domain

Tmem110 transmembrane protein 110

Tmem115 transmembrane protein 115

Tmem129 transmembrane protein 129

Tmem163 transmembrane protein 163

Tmem167 transmembrane protein 167

Tmem177 transmembrane protein 177

Tmem18 transmembrane protein 18

Tmem194 transmembrane protein 194 isoform 1

Tmem2 transmembrane protein 2

Tmem201 transmembrane protein 201 isoform b

Tmem214 hypothetical protein LOC68796

Tmem231 hypothetical protein LOC234740

Tmem30a transmembrane protein $30 \mathrm{~A}$

Tmem41a transmembrane protein $41 \mathrm{a}$

Tmem56 transmembrane protein 56

Tmem65 transmembrane protein 65

Tmem72 hypothetical protein LOC319776

Tmie integral membrane protein TMIE

Tmprss11f transmembrane protease serine $11 \mathrm{f}$

Tmprss2 transmembrane protease serine 2

Tmtc3 transmembrane and tetratricopeptide repeat

Tmx4 thioredoxin domain containing 13

Tnfaip3 tumor necrosis factor alpha-induced protein 3

Tnfaip8/3 tumor necrosis factor alpha-induced protein

Tnfrsf1b tumor necrosis factor receptor superfamily

Tnfsf18 tumor necrosis factor (ligand) superfamily

Tnrc6a trinucleotide repeat containing 6A

Tnrc6b trinucleotide repeat containing $6 \mathrm{~b}$ isoform 2

Tnrc6c trinucleotide repeat containing 6C

Tob2 transducer of ERBB2 2

Tomm70a translocase of outer mitochondrial membrane 70

Tor1aip2 torsin A interacting protein 2

Tor1aip2 interferon alpha responsive

Tpp1 tripeptidyl-peptidase I

Tppp tubulin polymerization-promoting protein 
Trabd TraB domain containing

Traf4 Tnf receptor associated factor 4

Tram1l1 translocation associated membrane protein 1-like

Trappc1 trafficking protein particle complex 1

Trem1 triggering receptor expressed on myeloid cells

Trhde TRH-degrading enzyme

Trib1 tribbles homolog 1

Trib2 tribbles homolog 2

Trim16 tripartite motif protein 16

Trim25 tripartite motif protein 25

Trim26 tripartite motif protein 26 isoform a

Trim41 tripartite motif-containing 41

Trim42 tripartite motif-containing 42

Trim6 tripartite motif protein 6

Trim66 tripartite motif-containing 66

Trim71 tripartite motif-containing 71

Trip13 thyroid hormone receptor interactor 13

Trmt11 RIKEN cDNA 2410075D05

Trove2 TROVE domain family member 2

Trp53bp1 transformation related protein 53 binding

Trp53inp1 transformation related protein 53 inducible

Trp73 transformation related protein 73

Trpc7 transient receptor potential cation channel

Trpm6 transient receptor potential cation channel

Tsc1 tuberous sclerosis 1

Tsc22d 2 TSC22 domain family 2

Tshr thyroid stimulating hormone receptor isoform 2

Tspan13 tetraspanin 13

Tspan18 tetraspanin 18

Tspan2 tetraspan 2

Tspan31 tetraspanin 31

Tspan 5 tetraspanin 5

Ttc19 tetratricopeptide repeat domain 19 isoform 2

Ttc39b hypothetical protein LOC69863

Ttc9c tetratricopeptide repeat domain 9C

Ttll4 tubulin tyrosine ligase-like family member 4

Tuba1b tubulin alpha 1B

Tuba4a tubulin alpha 4

Tubb3 tubulin beta 3 
Tusc2 tumor suppressor candidate 2

TxIna taxilin alpha

Txn2 thioredoxin 2

Tyk2 tyrosine kinase 2

Ubash3a ubiquitin associated and $\mathrm{SH}$ domain containing

Ube2g2 ubiquitin-conjugating enzyme E2G 2

Ube2j1 ubiquitin-conjugating enzyme E2 J1

Ube2q1 ubiquitin-conjugating enzyme E2Q

Ubfd1 ubiquitin family domain containing 1

Ubn2 hypothetical protein LOC320538

Ubox5 $U$ box domain containing 5

Ubxn10 UBX domain containing 3

Ubxn2b hypothetical protein LOC68053

Ubxn4 UBX domain containing 2

Ufm1 ubiquitin-fold modifier 1

Ugcg UDP-glucose ceramide glucosyltransferase

Uggt1 UDP-glucose ceramide glucosyltransferase-like 1

Uhrf1bp1 UHRF1 (ICBP90) binding protein 1

Uhrf2 ubiquitin-like containing PHD and RING finger

Ulk2 Unc-51 like kinase 2

Unc5a netrin receptor Unc5h1

Unc5d netrin receptor Unc5h4

Unc93a unc-93 homolog A

Urm1 ubiquitin related modifier 1 homolog

Usp12 ubiquitin specific peptidase 12

Usp20 ubiquitin specific peptidase 20

Usp21 ubiquitin-specific protease 21

Usp32 ubiquitin specific protease 32

Usp38 ubiquitin specific peptidase 38

Usp45 ubiquitin specific protease 45

Usp49 ubiquitin specific protease 49 homolog

Usp9x ubiquitin specific protease $9 \times$ chromosome

Uspl1 ubiquitin specific peptidase like 1 isoform $D$

Utrn utrophin

Vapa vesicle-associated membrane protein associated

Vash2 vasohibin 2

Vps25 vacuolar protein sorting 25

Vps26a vacuolar protein sorting 26 isoform a

Vps26b vacuolar protein sorting 26 homolog $B$ 
Vps37b vacuolar protein sorting 37B

Vsnl1 visinin-like 1

Vwa1 von Willebrand factor A domain-related protein

Wasl Wiskott-Aldrich syndrome-like

Wbscr22 Williams-Beuren syndrome critical region protein

Wdr26 WD repeat domain 26

Wdr37 WD repeat domain 37 isoform $b$

Wdr82 WD repeat domain containing 82

Wfdc16 WAP four-disulfide core domain 16

Whsc1l1 Wolf-Hirschhorn syndrome candidate 1-like 1

Wipi1 WD repeat domain phosphoinositide interacting

Wnt1 wingless-related MMTV integration site 1

Wnt3a wingless-related MMTV integration site 3A

Wnt9a wingless-type MMTV integration site 9A

Wnt9b wingless-type MMTV integration site 9B

Wtap Wilms' tumour 1-associating protein isoform a

Wwp1 WW domain-containing protein 1

X99384 paladin

Xk Kell blood group precursor (McLeod phenotype)

Xkr8 X Kell blood group precursor related family

Xpo7 exportin 7

Xpot exportin tRNA (nuclear export receptor for

Xrn1 5'-3' exoribonuclease 1

Yeats4 YEATS domain containing 4

Yod1 HIV-induced protein-7-like protease

Ypel2 yippee-like 2

Ythdf3 YTH domain family 3

Zbed3 zinc finger BED domain containing 3

Zbtb16 zinc finger and BTB domain containing 16

Zbtb26 zinc finger and BTB domain containing 26

Zbtb33 kaiso protein

Zbtb39 zinc finger and BTB domain containing 39

Zbtb7b zinc finger and BTB domain containing 7B

Zc3h11a zinc finger $\mathrm{CCCH}$ type containing $11 \mathrm{~A}$

Zc3h3 zinc finger $\mathrm{CCCH}$ type containing 3

Zc3h6 zinc finger CCCH type containing 6

Zc3hav1l zinc finger CCCH-type antiviral 1-like

Zcchc11 zinc finger CCHC domain containing 11

Zcchc3 zinc finger CCHC domain containing 3 
Zcchc5 zinc finger $\mathrm{CCHC}$ domain containing 5

Zdhhc12 zinc finger DHHC domain containing 12 isoform

Zdhhc21 zinc finger DHHC domain containing 21

Zdhhc25 zinc finger DHHC domain containing 25

Zdhhc3 zinc finger DHHC domain containing 3

Zfhx4 zinc finger homeodomain 4

Zfp11 zinc finger protein 11

Zfp113 zinc finger protein 113

Zfp161 zinc finger protein 161

Zfp2 zinc finger protein 2

Zfp202 zinc finger protein 202

Zfp248 zinc finger protein 248

Zfp275 Zinc finger protein 275

Zfp276 zinc finger protein 276

Zfp280b zinc finger protein 280b

Zfp282 zinc finger protein 282

Zfp318 zinc finger protein 318 isoform 2

Zfp334 zinc finger protein 334

Zfp341 zinc finger protein 341

Zfp354a zinc finger protein 354A

Zfp354b zinc finger protein 354B

Zfp362 zinc finger protein 362

Zfp36l1 zinc finger protein $36 \mathrm{C} 3 \mathrm{H}$ type-like 1

Zfp444 zinc finger protein 444

Zfp462 zinc finger protein 462

Zfp512 zinc finger protein 512

Zfp516 zinc finger protein 516

Zfp526 zinc finger protein 526

Zfp583 zinc finger protein 583

Zfp593 zinc finger protein 593

Zfp597 zinc finger protein 597

Zfp617 zinc finger protein 617

Zfp644 zinc finger motif enhancer binding protein 2

Zfp655 zinc finger protein 655 isoform b

Zfp664 zinc finger protein 664

Zfp668 zinc finger protein 668

Zfp689 zinc finger protein HIT-39

Zfp697 zinc finger protein 697

Zfp709 zinc finger protein 14 
Zfp710 zinc finger protein 710

Zfp740 zinc finger protein 740

Zfp748 zinc finger protein 748 isoform 1

Zfp759 zinc finger protein 759

Zfp9 zinc finger protein 9

Zfp92 zinc finger protein 92

Zfyve16 zinc finger FYVE domain containing 16

Zfyve26 zinc finger FYVE domain containing 26

Zik1 zinc finger protein interacting with $\mathrm{K}$ protein

Zkscan2 zinc finger with KRAB and SCAN domains 2

Zmat4 zinc finger matrin type 4

Zmym5 zinc finger MYM-type 5

Zscan10 zinc finger and SCAN domains 10

Zswim4 zinc finger SWIM domain containing 4

Zswim5 zinc finger SWIM domain containing 5 
ilable at http://www.mirz.unibas.ch/EIMMо3/. 